\title{
Exercise as a complementary therapy for depression: a systematic review and meta-analysis of randomized controlled trials and bioinformatics exploration gene network model .
}

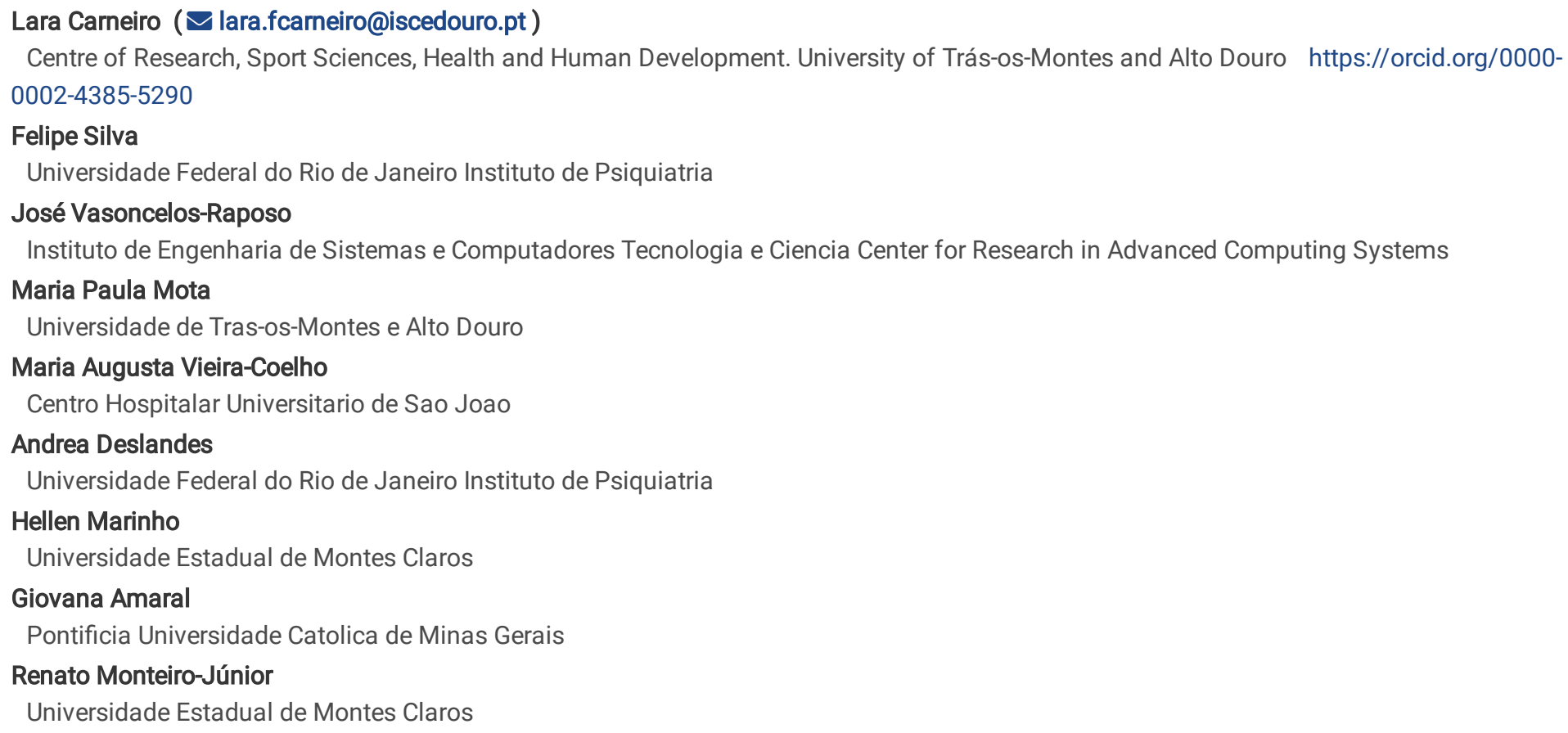

Research article

Keywords: Depression, exercise, pharmacological, treatment, dose-response

Posted Date: June 10th, 2020

DOI: https://doi.org/10.21203/rs.3.rs-24600/v1

License: (c) (i) This work is licensed under a Creative Commons Attribution 4.0 International License. Read Full License 


\section{Abstract}

Background The effect of pharmacological treatment (PT), exercise treatment (ET), and both in depressive symptoms remains a matter of debate.

The present study aimed to clarify (1) the effect of ET as a monotherapy or complementary treatment of pharmacological therapy; (2) changes in the dose-response for different exercise prescription characteristics; and 3) hypothesizing about the gene network model of exercise effects on depression.

Methods We sought Randomized Controlled Trials (RCT) addressing the effects of exercise on depressive patients, published in peer-reviewed journals between 2003-2019 in Scopus, Cochrane, Pubmed/Medline, ISI Web of Knowledge and APA PsycNET databases. Standardized mean difference (SMD) was calculated considering the mean difference on depression scales (pre and post-intervention) and pooled standard deviation for each intention-to-treat in each study. For the gene network model of exercise on depression an in silico analyses were used.

Results We found 1,165 articles and selected 15 studies to this meta-analysis. RCTs with different ET and PT prescriptions were examined using the delta (pre and post-intervention) of a validated depression scale compared to the control group in different treatment conditions.

Standardized mean differences and confidence intervals (SMD, 95\% Cl) were found for ET with or without PT group (-0.45, -0.62 to -0.29$)$, ET and PT group $(-0.70,-1.00$ to -0.40$)$, and only ET group $(-0.39,-0.57$ to -0.21$)$. The subgroup analyses showed a moderate and large SMD in favour of resistance training $(-0.67,-1.22$ to -0.12$)$, aerobic exercise $60-80 \%$ of maximum heart rate intensity $(-0.56,-0.89$ to -0.23$), 20$ to 30 minutes $(-0.59,-0.88$ to -0.31$)$ or 45 to 60 minutes $(-0.55,-0.81$ to -0.29$)$ duration per session, and seven times per week $(-0.75,-1.30$ to -0.20$)$.

Conclusion ET plus PT showed a better effect on reducing depressive symptoms, and the exercise prescription that shows the best doseresponse in subgroup analyses can be the target for ET in depression. Also, the genes network model can withstand the effect of exercise in the depressive symptoms decrease according to an interaction between BDNF and inflammation. More well-designed studies are needed to confirm our findings.

\section{Highlights}

- Exercise is an effective coadjuvant therapy for depression.

- Long exercise protocols seem more effective than adherence and benefit elderly the most.

- Gene network model showed biomarkers modulated by exercise.

\section{Background}

Major depressive disorder (MDD) is a worldwide mental health concern leading to severe morbidity and increased mortality risk [1]. Patients with depression have a twentyfold greater risk of suicide compared to the general population [2]. Several countries have conducted epidemiological surveys, and the high prevalence and persistence of MDD confirm that this is a worldwide disorder of utmost importance [3]. The years lived with disability (YLDs) per 1000 people with depression disease are more than five times higher than the existing global mean burden of disease estimates [4].

Regardless of the research growth during the last decade, no major advances have occurred in the treatment of depression [5]. Although pharmacological therapy (PT) is the current gold standard for the treatment of depression, this treatment may need a combination of therapies such as antidepressants, antipsychotics, electroconvulsive therapy and psychotherapy [6]. Thus, more investment is required to search for effective adjuvant therapies to enhance the treatment of depressive disorders. In recent years, systematic reviews and meta-analyses evidenced that physical activity and exercise have been improving depressive symptoms and benefit physical and mental health. Therefore, clinical guidelines have included exercise as a possible first-line intervention to the treatment of depressive symptoms [7].

Current reviews have been showing different methodologies and results when investigating the evidence that exercise treatment (ET) has a beneficial effect on reducing depressive symptoms [8-10]. Ekkekakis [11] suggests a carefully scrutiny on several grounds in these designstudies, hence aiming to eliminate these biases. Due to the potential inappropriate selection criteria applied, results could lead to misleading interpretations. Other reviews were published to set a consensual exercise prescription and recommend essential variables in the treatment of patients with depression [12-14].

Although these reviews have been helpful, there is an incomplete understanding of the effect of ET with and without PT in the depression treatment. While the impact of exercise interventions among subjects with depression has been explored, the optimal dose that might prove useful remains non-consensual. Also, studies with bioinformatics analyses [15] in an attempt to elucidate gene network models and 
biomarkers involved in the depression treatment with exercise are still lacking. Hypothetically, this analysis could be a reliable and straightforward form to study how exercise could decrease depressive symptoms. Different from the traditional published meta-analyses, the current study was structured with a robust methodological criterion to select and analyse the effect of ET with or without PT and bioinformatics data in depressive symptoms and exercise. Therefore, the current study intended to elucidate (1) the effect of ET with and without PT for depression; (2) changes in the dose-response of different exercise prescriptions, intervention and control group characteristics, and 3) a hypothesis about the gene network model in silico analyses to clarify how exercise could reduce depressive symptoms.

\section{Methods}

The registration of this systematic review and meta-analysis has been approved in the International Prospective Register of Systematic Reviews (PROSPERO) with the protocol number CRD42019122638. We followed the PICOS strategy [16] and the Preferred Reporting Items for Systematic Reviews and Meta-Analyses: The PRISMA Statement [17]. The recommendations of the Cochrane Collaboration [18] were used as a complementary guide.

\subsection{Eligibility criteria}

The acronym PICOS was primarily established to retrieve studies, as follow: depressive patients ( $\mathrm{P}$ - participants), exercise (I - intervention), control group ( $\mathrm{C}$ - comparison), depressive symptoms amelioration ( $\mathrm{O}$ - outcome) and randomized controlled trials (RCTs) ( $\mathrm{S}$ - study design).

\subsubsection{Types of studies}

RCTs conducted between 2003-2019 were selected, whether exercise was beneficial or not in the treatment of depression. To reduce the risk of bias, conference proceedings and unpublished studies were not used [19], and no restrictions on the language of the studies were applied.

\subsubsection{Participants}

Males and females aged 18 and over (with no upper age limit) with clinical depression as defined by the Diagnostic or Statistical Manual of Mental Disorders, Fourth Edition (DSM-IV), International Statistical Classification of Diseases and Related Health Problems 10th Revision (ICD10) criteria or other validated depression scale. Depression encompassing a co-morbid illness such as diabetes (insulin-dependent), cancer, multiple sclerosis, cardiovascular disease and mixed psychiatric diagnosis was not included.

\subsubsection{Interventions}

Intervention group had as ET aerobic or resistance training programs or a combination of both, in addition to PT, or not. In control group, we analysed interventions, such as pharmacotherapy, psychotherapy, another exercise program or no intervention (placebo or wait-list control). Considering exercise parameters, we included studies in accordance with the following data criteria: minutes per session, duration of intervention (week), frequency (days per week), adherence (percentage of finishers) and the amount of energy expended and measured in kilocalories per week ( $\mathrm{Kcal} /$ week), or the percentage of maximum heart rate $\left(\% \mathrm{HR}_{\max }\right)$, or the percentage of heart rate reserve (\%HRR), or the percentage of maximal oxygen uptake $\left(\mathrm{VO}_{2 \mathrm{max}}\right)$, or the percentage of maximal oxygen uptake reserve $\left(\mathrm{VO}_{2 \text { reseve }}\right)$, or metabolic equivalent (METs).

\subsubsection{Outcomes}

Pre and post-intervention scores of validated depression-rating scales were analysed. Studies that measured outcomes immediately before and after a single exercise session were not included.

\subsubsection{Information sources}

We conducted searches on PubMed/MedLine, Scopus, ISI Web of Knowledge, Cochrane Trials and APA PsycNET databases. No filters were used to search any of the databases to conduct the broadest search and reduce the risk of bias [19].

\subsubsection{Search}

Medical Subject Headings (MeSH) and search-indexed descriptors were used to refine data search [20]. Three thematic word groups with $\mathrm{MeSH}$ terms were used to conduct the searches. Within each group, the terms were combined using the Boolean operator OR and interaction between sets using the operator AND to form a phrase. Searches were conducted in April 2019 using the following terms: ("depressive disorder" or "unipolar depression" or "major depressive disorder") AND ("exercise" or "exercise programs") AND ("clinical trial" or "randomized controlled trial"). 


\subsubsection{Study selection and data collection process}

Studies were screened and data independently extracted by two researchers. Another researcher was requested to confirm the eligibility of the identified studies. Identified studies were tabulated on a worksheet (Microsoft Corporation, Redmond, USA) to confirm if they met the eligibility criteria. All their titles and abstracts were screened and the full-text articles were assessed for potential inclusion by the main investigator. Studies were included in the qualitative synthesis after the exclusion of a comprehensive text review. We identified all studies that presented a high risk of bias for the finish - selection process and finally, we included all quality studies into a quantitative synthesis to perform the metaanalysis.

\subsubsection{Appraisal of methodological quality}

The quality of the selected studies was appraised using the Delphi-list [21]. We analysed randomized controlled trials (RCT) through: randomization, allocation concealment, baseline comparability, eligibility criteria, blinding, descriptive measures for the primary outcome and intention-to-treat analysis [22]. In this study, except double-blinding, which is not applicable within the framework of trials involving physical exercises, all these features were taken into account by the qualifying examination. Two researchers independently calculated an overall quality score of the Delphi items that scored positive and discussed them to achieve consensus. Studies selected did exhibit weaknesses concerning some criteria and these deficiencies were taken into consideration and explained in the results and discussion sections.

\subsection{Data selection}

We selected the following characteristics in all studies: 1 ) total number and age of each group; 2) depression-rating scales used to the diagnosis; 3) type of intervention and other exercise parameters mentioned in the section 2.1.3; 4) pre (M1) and post (M2) intervention depression-rating scales scores ( $n$, means and standard deviation) to calculate the effect size (ES) of intervention and control groups. The mean difference $(M D=M 2-M 1)$ and the pooled standard deviation $\left(S_{\text {pooled }}=\sqrt{ }\left(\mathrm{SD}_{M 2}{ }^{2}\left(n_{M 2}-1\right)+S_{M 1}{ }^{2}\left(n_{M 1}-1\right)\right) /\left(n_{M 2}+n_{M 1}-2\right)\right)$ of each group in all studies were calculated to ES analyses [23]. Regarding pre-selected articles that did not present the necessary data in the text, values were requested to the authors by e-mail.

\subsection{Risk of bias}

The risk of bias was assessed using qualitative analysis for each included study, and each risk of bias item presented on the Delphi-list. This scale provides a quality assessment of RCT studies, and the high quality is defined as achieving over $50 \%$ of the maximum attainable score, meaning five or more criteria met on the Delphi-list [21]. To analyse the risk of publication bias, we used funnel plot visual inspection. The risk among studies was assessed using the results of heterogeneity within the forest plot. Heterogeneity was measured using the $\mathrm{T}^{2}, \mathrm{X}^{2}$, and $\mathrm{I}^{2}$ tests. In the $T^{2}$ test, $T^{2}>1$ suggests the presence of substantial statistical heterogeneity. If the $X^{2}$ value is statistically significant $(p<0.05)$, there is also evidence of heterogeneity. In the $I^{2}$ test analysis, the percentage of the variance attributed to the heterogeneity of the study ranges from low $\left(25 \%<1^{2}<50 \%\right)$ to moderate $\left(50 \%<1^{2}<75 \%\right)$ to high $\left(1^{2}>75 \%\right)[19]$.

\subsection{Summary measures}

Analyses were performed considering two different groups, experimental and control. The main analysis was related to the additional effect of ET with or without PT for depression. Also, we included the effect of subgroup analyses among different exercise prescriptions, intervention, and control group characteristics encompassed in the selected studies. Control group data were replicated and compared with the different intervention groups in their studies to comparison analysis in this meta-analysis For multiple comparison groups in the same study, control group had the sample divided based on the number of groups that existed for comparison [24]. This was to maintain control group correct sample size and allocate the right weight to groups with more subjects.

Standardized mean difference (SMD) was calculated considering the mean difference on depression scales (pre and post-intervention) and pooled standard deviation for each intention-to-treat in each study. This outcome was reported on different validated scales. Consequently, the SMD in this review was calculated based on the random-effect model with $95 \%$ confidence intervals (Cl: $95 \%$ ), including the assumption of heterogeneity of the studies and their participants. Analyses of forest and funnel plots were performed using the Review Manager (RevMan) Version 5.3 software (Copenhagen: The Nordic Cochrane Centre, The Cochrane Collaboration, 2014).

\subsection{The Gene Network Model in silico analyses}

The GeneCards database (https://www.genecards.org) was used to explore proteins-related genes associated with exercise and depression.

Keywords "exercise" OR "physical activity" AND "major depression" were inserted into database to identify genes associated with the subject. GeneCards organizes genes search according to a qualitative score. A score above 10 is acceptable to indicate the gene is known to be 
functional (see https://www.genecards.org/Guide/GeneCard). To increase the power of the analysis, genes with score 30 or above were retrieved from GeneCards as follows: interleukin 6 (IL-6, score 42.60), tumour necrosis factor (TNF, score 42.44), solute carrier family 6 member 4 (SLC6A4, score 40.72), 5-hydroxytryptamine receptor 2A (HTR2A, score 37.06), tryptophan hydroxylase 2 (TPH2, score 36.12), insulin (INS, score 35.63), brain-derived neurotrophic factor (BDNF, score 32.05) and apolipoprotein E (APOE, score 30.53). Genes aforementioned were inserted into the String database (https://string-db.org), which permits exploring genes and their encoded protein interactions as a network. String uses data mining to find published articles which have investigated direct or indirectly targeted protein interactions in different species. According to the interactions found, we can suppose that exercise could result similar interactions, independently of the results from metaanalysis. Our interaction exploration was performed considering Homo Sapiens as the studied specie.

\section{Results}

\subsection{Study selection and characteristics}

A total of 1,165 articles was found (PubMed $=235$; Cochrane $=334 ;$ Scopus $=438$; ISI Web of Knowledge = 133; APA PsycNET = 25). After applying the exclusion criteria for eligibility, a total of 17 studies were subjected to a qualitative analysis. After this, 15 studies were included, and two studies were excluded from the meta-analysis. Figure 1 shows the screening flow used in the study selection.

This review shows 17 studies selected that met the quality assessment score based on the Delphi-list (Table 1). Regarding the RCT studies evaluated, scores ranged between 3 and 7 points (mean $=5.5$ points). Two studies $[25,26]$ scored 3 , indicating unsatisfactory quality, and therefore, were excluded.

Table 1 - Evaluation of the methodological quality of the RCT studies by the Delphi-list. 


\begin{tabular}{|c|c|c|c|c|c|c|c|c|c|c|}
\hline \begin{tabular}{|c|} 
Randomized \\
Clinical \\
Trials
\end{tabular} & $\begin{array}{c}\text { Was a method } \\
\text { of } \\
\text { randomization } \\
\text { performed? }\end{array}$ & \begin{tabular}{|l|} 
Was the \\
treatment \\
allocation \\
concealed?
\end{tabular} & \begin{tabular}{|} 
Were the groups \\
similar at \\
baseline \\
regarding the \\
most important \\
prognostic \\
indicators?
\end{tabular} & $\begin{array}{l}\text { Were the } \\
\text { eligibility } \\
\text { criteria } \\
\text { specified? }\end{array}$ & $\begin{array}{l}\text { Was the } \\
\text { outcome } \\
\text { assessor- } \\
\text { blinded? }\end{array}$ & $\begin{array}{l}\text { Was the } \\
\text { care } \\
\text { provider } \\
\text { blinded? }\end{array}$ & $\begin{array}{c}\text { Was the } \\
\text { patient } \\
\text { blinded? }\end{array}$ & $\begin{array}{c}\text { Were point estimates and } \\
\text { measures of variability } \\
\text { presented for the primary } \\
\text { outcome } \\
\text { measures? }\end{array}$ & $\begin{array}{c}\text { Did the } \\
\text { analysis } \\
\text { include an } \\
\text { intention-to- } \\
\text { treat } \\
\text { analysis? }\end{array}$ & $\begin{array}{l}\text { Quality } \\
\text { score }\end{array}$ \\
\hline $\begin{array}{c}\text { Buschert, } \\
\text { Prochazka } \\
\text { [27] }\end{array}$ & $\mathrm{Y}$ & $\mathrm{Y}$ & $\mathrm{N}$ & $\mathrm{Y}$ & ? & ? & ? & $\mathrm{Y}$ & $\mathrm{Y}$ & 5 \\
\hline \begin{tabular}{|c|} 
Carneiro, \\
Fonseca [28]
\end{tabular} & $\mathrm{Y}$ & $\mathrm{Y}$ & $\mathrm{Y}$ & $\mathrm{Y}$ & $\mathrm{N}$ & $\mathrm{N}$ & $\mathrm{N}$ & $\mathrm{Y}$ & $\mathrm{Y}$ & 6 \\
\hline \begin{tabular}{|l|} 
de la Cerda, \\
Cervello [26]
\end{tabular} & $\mathbf{N}$ & $\mathbf{N}$ & $\mathbf{N}$ & $Y$ & $\mathbf{N}$ & $\mathbf{N}$ & $\mathbf{N}$ & $Y$ & $\mathrm{Y}$ & 3\# \\
\hline $\begin{array}{c}\text { Cheung and } \\
\text { Lee [29] }\end{array}$ & $\mathrm{Y}$ & $\mathrm{Y}$ & $\mathrm{Y}$ & $\mathrm{Y}$ & $\mathrm{Y}$ & $\mathrm{N}$ & $\mathrm{N}$ & $\mathrm{Y}$ & $\mathrm{Y}$ & 7 \\
\hline $\begin{array}{c}\text { Dunn, } \\
\text { Trivedi [30] }\end{array}$ & $\mathrm{Y}$ & $\mathrm{Y}$ & $\mathrm{Y}$ & $\mathrm{Y}$ & $?$ & $\mathrm{Y}$ & $\mathrm{N}$ & $\mathrm{Y}$ & $\mathrm{Y}$ & 7 \\
\hline $\begin{array}{c}\text { Hallgren, } \\
\text { Kraepelien } \\
{[31]}\end{array}$ & $\mathrm{Y}$ & $\mathrm{Y}$ & ? & $\mathrm{Y}$ & ? & ? & ? & $\mathrm{Y}$ & $\mathrm{Y}$ & 5 \\
\hline $\begin{array}{c}\text { Hoffman, } \\
\text { Blumenthal } \\
{[32]}\end{array}$ & $\mathrm{Y}$ & $?$ & $?$ & $\mathrm{Y}$ & $\mathrm{Y}$ & $\mathrm{Y}$ & ? & $\mathrm{Y}$ & $\mathrm{Y}$ & 6 \\
\hline \begin{tabular}{|c|} 
Kerling, \\
Tegtbur [33]
\end{tabular} & $\mathrm{Y}$ & $\mathrm{Y}$ & $\mathrm{Y}$ & $\mathrm{Y}$ & $?$ & $?$ & ? & $\mathrm{Y}$ & $\mathrm{Y}$ & 6 \\
\hline $\begin{array}{c}\text { Knubben, } \\
\text { Reischies } \\
\text { [34] }\end{array}$ & $\mathrm{Y}$ & $\mathrm{Y}$ & $?$ & $\mathrm{Y}$ & $?$ & $?$ & $\mathrm{~N}$ & $\mathrm{Y}$ & $\mathrm{Y}$ & 5 \\
\hline $\begin{array}{c}\text { Legrand and } \\
\text { Neff [36] }\end{array}$ & $\mathrm{Y}$ & $\mathrm{Y}$ & $\mathrm{Y}$ & $\mathrm{Y}$ & ? & ? & ? & $\mathrm{Y}$ & $\mathrm{Y}$ & 6 \\
\hline $\begin{array}{c}\text { Belvederi } \\
\text { Murri, } \\
\text { Amore [37] }\end{array}$ & $\mathrm{Y}$ & $\mathrm{Y}$ & ? & $\mathrm{Y}$ & ? & ? & ? & $\mathrm{Y}$ & $\mathrm{Y}$ & 5 \\
\hline $\begin{array}{c}\text { Olson, Brush } \\
{[38]}\end{array}$ & $\mathrm{Y}$ & $\mathrm{Y}$ & $\mathrm{Y}$ & $\mathrm{Y}$ & $?$ & $?$ & ? & $\mathrm{Y}$ & $\mathrm{Y}$ & 6 \\
\hline $\begin{array}{c}\text { Roy, } \\
\text { Govindan } \\
\text { [39] }\end{array}$ & $\mathrm{Y}$ & $\mathrm{N}$ & $\mathrm{Y}$ & $\mathrm{Y}$ & $\mathrm{N}$ & $\mathrm{N}$ & $\mathrm{N}$ & $\mathrm{Y}$ & $\mathrm{Y}$ & 5 \\
\hline \begin{tabular}{|c|} 
Schuch, \\
Vasconcelos- \\
Moreno [25]
\end{tabular} & $\mathrm{Y}$ & $?$ & $?$ & $Y$ & $?$ & $?$ & $?$ & $\mathbf{N}$ & $Y$ & 3\# \\
\hline $\begin{array}{c}\text { Siqueira, } \\
\text { Valiengo } \\
{[40]}\end{array}$ & $\mathrm{Y}$ & $\mathrm{Y}$ & $\mathrm{Y}$ & $\mathrm{Y}$ & $?$ & $\mathrm{Y}$ & $?$ & $\mathrm{Y}$ & $\mathrm{Y}$ & 7 \\
\hline $\begin{array}{c}\text { Strom, } \\
\text { Uckelstam } \\
\text { [41] }\end{array}$ & $\mathrm{Y}$ & $\mathrm{Y}$ & ? & $\mathrm{Y}$ & ? & ? & ? & $\mathrm{Y}$ & $\mathrm{Y}$ & 5 \\
\hline
\end{tabular}

Y: yes; N: no; ?: Don't know; \#: excluded

\subsection{Summary of studies}

The 15 studies included in the meta-analysis provided a total sample size of 1,532 individuals, with a mean age 44 years, which ranged from 21 to 75 years. The mean percentual of females' sample was $66.5 \%$ and varied between 30 and 100\%. Investigations occurred in different countries, expanding different personal characteristics in the present study. Co-morbidities were not found among the participants. In all selected articles, interventions with aerobic, resistance training, or a combination of both, and validated depression-scales were used, and other characteristics of the studies are described in Table 2.

Table 2 - Characteristics of selected studies. 


\begin{tabular}{|c|c|c|c|c|c|c|c|c|c|c|c|c|c|}
\hline RCT study & Groups & $\mathbf{N}$ & Sex, F \% & $\begin{array}{c}\text { Age, } \\
\text { years }\end{array}$ & $\begin{array}{c}\text { Main } \\
\text { outcome }\end{array}$ & $\begin{array}{c}\text { Pre- } \\
\text { intervention } \\
\text { outcome }\end{array}$ & $\begin{array}{c}\text { Post- } \\
\text { intervention } \\
\text { outcome }\end{array}$ & Intervention & $\begin{array}{c}\text { Minutes } \\
\text { per } \\
\text { session }\end{array}$ & $\begin{array}{c}\text { Time of } \\
\text { intervention } \\
\text { week }\end{array}$ & $\begin{array}{l}\text { Frequency, } \\
\text { day/week }\end{array}$ & Intensity & Adherence \\
\hline \multirow[t]{2}{*}{$\begin{array}{c}\text { Buschert, } \\
\text { Prochazka } \\
\text { [27] }\end{array}$} & $\begin{array}{l}\text { Control } \\
\text { group }\end{array}$ & 15 & 66.67 & $\begin{array}{l}47.47 \\
(8.47)\end{array}$ & HDRS-7 & $9.67 \pm 4.21$ & $6.44 \pm 3.43$ & $\begin{array}{c}\text { TAU + } \\
\text { occupational } \\
\text { or art } \\
\text { therapy }\end{array}$ & 30 & 3 to 4 & 2 to 3 & not reported & $\begin{array}{c}\text { not } \\
\text { reported }\end{array}$ \\
\hline & $\begin{array}{l}\text { Intervention } \\
\text { group }\end{array}$ & 15 & 60 & $\begin{array}{l}47.27 \\
(6.84)\end{array}$ & HDRS-7 & $11.00 \pm 3.42$ & $7.20 \pm 3.88$ & $\begin{array}{l}\text { Endurance } \\
\text { training }\end{array}$ & 30 & 3 to 4 & 2 to 3 & $\begin{array}{r}>85 \% \\
\text { HRmax }\end{array}$ & $\begin{array}{c}\text { not } \\
\text { reported }\end{array}$ \\
\hline \multirow{2}{*}{$\begin{array}{c}\text { Carneiro, } \\
\text { Fonseca } \\
{[28]}\end{array}$} & $\begin{array}{l}\text { Control } \\
\text { group }\end{array}$ & 10 & $\begin{array}{c}\text { not } \\
\text { reported }\end{array}$ & $\begin{array}{c}47.80 \\
(15.05)\end{array}$ & BDI-II & $46.10 \pm 11.52$ & $\begin{array}{c}49.40 \pm \\
16.72\end{array}$ & TAU & - & - & - & - & $\begin{array}{c}\text { not } \\
\text { reported }\end{array}$ \\
\hline & $\begin{array}{l}\text { Intervention } \\
\text { group }\end{array}$ & 9 & $\begin{array}{c}\text { not } \\
\text { reported }\end{array}$ & $\begin{array}{l}52.78 \\
(7.66)\end{array}$ & BDI-II & $45.56 \pm 9.65$ & $\begin{array}{c}34.89 \pm \\
10.56\end{array}$ & $\begin{array}{l}\text { Warm-up, } \\
\text { aerobics and } \\
\text { cool-down. }\end{array}$ & $45-50$ & 16 & 3 & $\begin{array}{c}\text { Average } \\
\text { number of } 4 \\
\text { months was } \\
65 \%, 73 \%, \\
74 \% \text { and } 76 \% \\
\text { HRmax, } \\
\text { respectively }\end{array}$ & $\begin{array}{c}\text { not } \\
\text { reported }\end{array}$ \\
\hline \multirow{2}{*}{$\begin{array}{l}\text { Cheung } \\
\text { and Lee } \\
\text { [29] }\end{array}$} & $\begin{array}{l}\text { Control } \\
\text { group }\end{array}$ & 17 & 70.6 & $\begin{array}{c}48.1 \\
(10.8)\end{array}$ & $\begin{array}{c}\text { HDRS- } \\
17\end{array}$ & $19.40 \pm 3.60$ & $\begin{array}{c}14.50 \pm \\
5.30\end{array}$ & TAU & - & - & - & - & $\begin{array}{c}\text { not } \\
\text { reported }\end{array}$ \\
\hline & $\begin{array}{l}\text { Intervention } \\
\text { group }\end{array}$ & 17 & 88.2 & $\begin{array}{c}47.4 \\
(11.2)\end{array}$ & $\begin{array}{c}\text { HDRS- } \\
17\end{array}$ & $18.5 \pm 3.20$ & $9.80 \pm 5.60$ & $\begin{array}{c}\text { TAU + } \\
\text { 1x/week } \\
\text { aerobic } \\
\text { session } \\
\text { under } \\
\text { supervision; } \\
\text { at least } \\
\text { 2x/week } \\
\text { aerobic } \\
\text { session } \\
\text { home } \\
\text { training }\end{array}$ & 60 & 12 & $\begin{array}{c}3 \mathrm{x} / \mathrm{w} \text { at } \\
\text { least }\end{array}$ & $>60 \%$ HRmax & $\begin{array}{c}52 \%, \\
82.2 \%, \\
\text { and } 38.9 \%\end{array}$ \\
\hline \multirow{5}{*}{$\begin{array}{l}\text { Dunn, } \\
\text { Trivedi } \\
\text { [30] }\end{array}$} & $\begin{array}{l}\text { Control } \\
\text { group }\end{array}$ & 13 & 62 & $\begin{array}{l}34.5 \\
(7.3)\end{array}$ & $\begin{array}{c}\text { HDRS- } \\
17\end{array}$ & $20.50 \pm 2.40$ & $\begin{array}{c}14.00 \pm \\
4.90\end{array}$ & $\begin{array}{l}\text { Stretching } \\
\text { exercise }\end{array}$ & 15 to 20 & 12 & 3 & not reported & $42 \%$ \\
\hline & $\begin{array}{l}\text { Intervention } \\
\text { LD/3 group }\end{array}$ & 16 & 81 & $\begin{array}{l}35.8 \\
(6.1)\end{array}$ & $\begin{array}{c}\text { HDRS- } \\
17\end{array}$ & $19.30 \pm 2.60$ & $\begin{array}{l}11.70 \pm \\
5.80\end{array}$ & $\begin{array}{c}\text { Aerobic LD, } \\
3 \text { days per } \\
\text { week }\end{array}$ & $>20$ & 12 & 3 & $\begin{array}{c}7 \\
\mathrm{kcal} / \mathrm{kg} / \mathrm{week} \\
\text { low dose }\end{array}$ & $78 \%$ \\
\hline & $\begin{array}{l}\text { Intervention } \\
\text { LD/5 group }\end{array}$ & 18 & 72 & $\begin{array}{l}37.7 \\
(5.1)\end{array}$ & $\begin{array}{c}\text { HDRS- } \\
17\end{array}$ & $19.20 \pm 2.30$ & $\begin{array}{l}12.80 \pm \\
5.00\end{array}$ & $\begin{array}{c}\text { Aerobic LD, } \\
5 \text { days per } \\
\text { week }\end{array}$ & $>20$ & 12 & 5 & $\begin{array}{c}7 \\
\mathrm{kcal} / \mathrm{kg} / \mathrm{week} \\
\text { low dose }\end{array}$ & $78 \%$ \\
\hline & $\begin{array}{c}\text { Intervention } \\
\text { PHD/3 } \\
\text { group }\end{array}$ & 17 & 76 & $\begin{array}{l}33.2 \\
(6.7)\end{array}$ & $\begin{array}{c}\text { HDRS- } \\
17\end{array}$ & $19.10 \pm 1.80$ & $9.00 \pm 3.60 \mathrm{~A}$ & $\begin{array}{c}\text { Aerobic PHD, } \\
3 \text { days per } \\
\text { week }\end{array}$ & $>20$ & 12 & 3 & $\begin{array}{c}17.5 \\
\mathrm{kcal} / \mathrm{kg} / \text { week; } \\
\text { public health } \\
\text { dose }\end{array}$ & $65 \%$ \\
\hline & $\begin{array}{c}\text { Intervention } \\
\text { PHD/5 } \\
\text { group }\end{array}$ & 16 & 81 & $37.9(6.3)$ & $\begin{array}{c}\text { HDRS- } \\
17\end{array}$ & $19.10 \pm 2.20$ & $\begin{array}{c}10.00 \pm A \\
5.50\end{array}$ & $\begin{array}{c}\text { Aerobic PHD, } \\
5 \text { days per } \\
\text { week }\end{array}$ & $>20$ & 12 & 5 & $\begin{array}{c}17.5 \\
\text { kcal/kg/week; } \\
\text { public health } \\
\text { dose }\end{array}$ & $65 \%$ \\
\hline \multirow[t]{2}{*}{$\begin{array}{c}\text { Hallgren, } \\
\text { Kraepelien } \\
\text { [31] }\end{array}$} & $\begin{array}{l}\text { Control } \\
\text { group }\end{array}$ & 232 & $\begin{array}{l}\text { not } \\
\text { reported r } \\
\text { to each } \\
\text { group }\end{array}$ & $\begin{array}{l}\text { not } \\
\text { reported } \\
\text { to each } \\
\text { group }\end{array}$ & MADRS & $20.90 \pm 7.50$ & $\begin{array}{c}13.80 \pm \\
8.90\end{array}$ & $\begin{array}{l}\text { TAU and } \\
\text { counselling } \\
\text { with a CBT }\end{array}$ & $\begin{array}{c}\text { not } \\
\text { reported }\end{array}$ & 12 & $\begin{array}{c}\text { not } \\
\text { reported }\end{array}$ & not reported & $30 \%$ \\
\hline & $\begin{array}{c}\text { Exercise } \\
\text { intervention } \\
\text { group }\end{array}$ & 249 & $\begin{array}{l}\text { not } \\
\text { reported r } \\
\text { to each } \\
\text { group }\end{array}$ & $\begin{array}{l}\text { not } \\
\text { reported } \\
\text { to each } \\
\text { group }\end{array}$ & MADRS & $22.20 \pm 6.80$ & $\begin{array}{r}11.30 \pm \\
7.90 \\
\\
\\
\\
b\end{array}$ & $\begin{array}{l}\text { Randomized } \\
\text { with three } \\
\text { conditions: } \\
\text { yoga, or } \\
\text { aerobics, or } \\
\text { aerobics + } \\
\text { strength + } \\
\text { balance class }\end{array}$ & 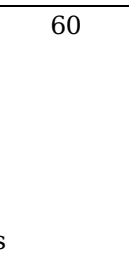 & 12 & 3 & $\begin{array}{c}\text { light, } \\
\text { moderate, } \\
\text { and vigorous } \\
\text { exercise }\end{array}$ & $33 \%$ \\
\hline \multirow{3}{*}{$\begin{array}{c}\text { Hoffman, } \\
\text { Blumenthal } \\
\text { [32] }\end{array}$} & Placebo & 49 & 77.5 & $\begin{array}{l}51.2 \\
(7.8)\end{array}$ & $\begin{array}{c}\text { HDRS- } \\
17\end{array}$ & $17.20 \pm 4.30$ & $\begin{array}{c}11.00 \pm \\
7.00\end{array}$ & Placebo pill & - & - & - & - & $86 \%$ \\
\hline & $\begin{array}{l}\text { Supervised } \\
\text { exercise }\end{array}$ & 51 & 76.5 & $\begin{array}{l}51.0 \\
(7.0)\end{array}$ & $\begin{array}{c}\text { HDRS- } \\
17\end{array}$ & $16.40 \pm 3.70$ & $9.20 \pm 6.10$ & $\begin{array}{c}\text { Aerobic } \\
\text { exercise } \\
\text { (treadmill) }\end{array}$ & $\begin{array}{c}\text { not } \\
\text { reported }\end{array}$ & 16 & 3 & $\begin{array}{l}70-85 \% \text { of } \\
\text { their HRR }\end{array}$ & $60.6 \%$ \\
\hline & $\begin{array}{l}\text { Home- } \\
\text { based }\end{array}$ & 53 & 73.6 & $\begin{array}{l}52.8 \\
(7.7)\end{array}$ & $\begin{array}{c}\text { HDRS- } \\
17\end{array}$ & $17.30 \pm 4.60$ & $\begin{array}{l}10.20 \pm \\
6.70\end{array}$ & $\begin{array}{l}\text { Aerobic } \\
\text { exercise }\end{array}$ & $\begin{array}{c}\text { not } \\
\text { reported }\end{array}$ & 16 & 3 & $\begin{array}{l}70-85 \% \text { of } \\
\text { their HRR }\end{array}$ & $60.6 \%$ \\
\hline
\end{tabular}

Page $7 / 20$ 
exercise

(outside)

\begin{tabular}{|c|c|c|c|c|c|c|c|c|c|c|c|c|c|}
\hline $\begin{array}{l}\text { Kerling, } \\
\text { Tegtbur } \\
\text { [33] }\end{array}$ & $\begin{array}{l}\text { Control } \\
\text { group }\end{array}$ & 20 & 30 & $\begin{array}{c}40.9 \\
(11.9)\end{array}$ & $\begin{array}{c}\text { BDI-II } \\
\text { and } \\
\text { MADRS }\end{array}$ & $\begin{array}{l}28.30 \pm \\
11.20 \\
24.50 \pm \\
10.30\end{array}$ & $\begin{array}{c}15.90 \pm \\
12.50 \\
16.40 \pm \\
9.40\end{array}$ & $\begin{array}{l}\text { TAU + CBT } \\
+ \text { workout } \\
\text { supervised in } \\
\text { the morning }\end{array}$ & 20 & 6 & 7 & light & $\begin{array}{c}\text { not } \\
\text { reported }\end{array}$ \\
\hline & $\begin{array}{l}\text { Intervention } \\
\text { group }\end{array}$ & 22 & 45 & $\begin{array}{l}44.2 \\
(8.5)\end{array}$ & $\begin{array}{c}\text { BDI-II } \\
\text { and } \\
\text { MADRS }\end{array}$ & $\begin{array}{c}29.40 \pm \\
10.90 \\
23.50 \pm 8.70\end{array}$ & $\begin{array}{c}13.40 \pm \\
13.20 \\
11.80 \pm \\
10.40\end{array}$ & $\begin{array}{c}\text { CBT + } \\
\text { Aerobic } \\
\text { workout }\end{array}$ & 45 & 6 & 3 & $\begin{array}{c}\text { moderate } \\
\text { intensity with } \\
\text { incremental } \\
\text { exercise }\end{array}$ & $90 \%$ \\
\hline \multirow{2}{*}{$\begin{array}{c}\text { Knubben, } \\
\text { Reischies } \\
\text { [34] }\end{array}$} & $\begin{array}{l}\text { Control } \\
\text { group }\end{array}$ & 18 & 55.55 & $50(13)$ & CES-D & $39.20 \pm 8.50$ & $\begin{array}{c}31.80 \pm \\
11.20\end{array}$ & $\begin{array}{l}\text { Low-intensity } \\
\text { stretching }\end{array}$ & 30 & 10 days & daily & not reported & $\begin{array}{c}\text { not } \\
\text { reported }\end{array}$ \\
\hline & $\begin{array}{l}\text { Intervention } \\
\text { group }\end{array}$ & 20 & 55.00 & 49 (13) & CES-D & $37.60 \pm 12.90$ & $\begin{array}{c}22.40 \pm \\
10.00\end{array}$ & $\begin{array}{c}\text { Interval } \\
\text { training on } \\
\text { treadmill }\end{array}$ & 30 & 10 days & daily & 80\% HRmax & $\begin{array}{c}\text { not } \\
\text { reported }\end{array}$ \\
\hline \multirow[t]{2}{*}{$\begin{array}{c}\text { Krogh, } \\
\text { Videbech } \\
\text { [35] }\end{array}$} & $\begin{array}{l}\text { Control } \\
\text { group }\end{array}$ & 59 & 62.7 & $\begin{array}{c}43.4 \\
(11.2)\end{array}$ & $\begin{array}{l}\text { HDRS- } \\
17 \text { and } \\
\text { BDI-II }\end{array}$ & $\begin{array}{l}18.60 \pm 4.00 \\
35.50 \pm 8.40\end{array}$ & $\begin{array}{c}10.50 \pm \\
6.40 ; \\
21.20 \pm \\
15.10\end{array}$ & $\begin{array}{c}\text { Social } \\
\text { interaction } \\
\text { and contact } \\
\text { with health } \\
\text { care } \\
\text { professionals } \\
\text { in an aerobic } \\
\text { exercise } \\
\text { group. }\end{array}$ & 45 & 9 & 3 & not reported & $\begin{array}{c}\text { not } \\
\text { reported }\end{array}$ \\
\hline & $\begin{array}{l}\text { Intervention } \\
\text { group }\end{array}$ & 56 & 71.4 & $\begin{array}{c}39.7 \\
(11.3)\end{array}$ & $\begin{array}{l}\text { HDRS- } \\
17 \text { and } \\
\text { BDI-II }\end{array}$ & $\begin{array}{l}19.20 \pm 4.70 \\
35.70 \pm 7.00\end{array}$ & $\begin{array}{c}11.30 \pm \\
6.60 \\
21.70 \pm \\
13.90\end{array}$ & $\begin{array}{c}\text { Aerobic } \\
\text { exercise on a } \\
\text { stationary } \\
\text { cycle } \\
\text { ergometer }\end{array}$ & 45 & 9 & 3 & $\begin{array}{c}4 \text { week } 65 \% \\
\text { HRR at least, } \\
\text { progressing } \\
\text { to } 70 \% \text { and } \\
80 \% \text { in } 3 \text { and } \\
4 \text { months }\end{array}$ & $66 \%$ \\
\hline \multirow{2}{*}{$\begin{array}{c}\text { Legrand } \\
\text { and Neff } \\
\text { [36] }\end{array}$} & $\begin{array}{l}\text { Control } \\
\text { group }\end{array}$ & 10 & 70 & $\begin{array}{c}49.1 \\
(16.5)\end{array}$ & BDI-II & $35.70 \pm 6.70$ & $\begin{array}{c}29.70 \pm \\
12.30\end{array}$ & TAU & - & - & - & - & $\begin{array}{c}\text { not } \\
\text { reported }\end{array}$ \\
\hline & $\begin{array}{l}\text { Aerobic } \\
\text { group }\end{array}$ & 14 & 64.3 & $\begin{array}{c}45.3 \\
(10.6)\end{array}$ & BDI-II & $36.10 \pm 5.90$ & $18.50 \pm 6.0$ & $\begin{array}{l}\text { Daily brisk } \\
\text { walking or } \\
\text { jogging }\end{array}$ & 30 & 10 days & daily & $\begin{array}{l}65-75 \% \\
\text { HRmax }\end{array}$ & $80 \%$ \\
\hline \multirow{3}{*}{$\begin{array}{c}\text { Belvederi } \\
\text { Murri, } \\
\text { Amore [37] }\end{array}$} & $\begin{array}{l}\text { Control } \\
\text { group }\end{array}$ & 42 & 76 & $\begin{array}{l}75.6 \\
(5.6)\end{array}$ & $\begin{array}{l}\text { HDRS- } \\
17\end{array}$ & $20.40 \pm 3.40$ & $\begin{array}{c}11.70 \pm \\
5.90\end{array}$ & TAU & - & - & - & - & $26 \%$ \\
\hline & $\begin{array}{l}\text { Sertraline } \\
\text { plus non- } \\
\text { progressive } \\
\text { activity } \\
\text { group }\end{array}$ & 37 & 68 & $\begin{array}{l}75.0 \\
(6.3)\end{array}$ & $\begin{array}{c}\text { HDRS- } \\
17\end{array}$ & $20.10 \pm 3.20$ & $8.50 \pm 4.50$ & $\begin{array}{c}\text { TAU + } \\
\text { Strength, } \\
\text { balance, } \\
\text { respiration } \\
\text { and motor } \\
\text { coordination }\end{array}$ & 60 & 24 & 3 & $\begin{array}{l}\text { 70\% HRmax } \\
\text { non- } \\
\text { progressive } \\
\text { intensity }\end{array}$ & $70 \%$ \\
\hline & $\begin{array}{l}\text { Sertraline } \\
\text { plus } \\
\text { progressive } \\
\text { activity } \\
\text { group }\end{array}$ & 42 & 69 & $\begin{array}{l}75.0 \\
(6.2)\end{array}$ & $\begin{array}{c}\text { HDRS- } \\
17\end{array}$ & $19.80 \pm 2.60$ & $7.10 \pm 4.20$ & $\begin{array}{c}\text { TAU + } \\
\text { Warm-up, } \\
\text { cycling, and } \\
\text { cool-down }\end{array}$ & 60 & 24 & 3 & $\begin{array}{l}\text { Start with } \\
60 \% \text { HRmax } \\
\text { for } \\
\text { progressive } \\
\text { intensity }\end{array}$ & $70 \%$ \\
\hline \multirow[t]{2}{*}{$\begin{array}{c}\text { Olson, } \\
\text { Brush [38] }\end{array}$} & $\begin{array}{l}\text { Control } \\
\text { group }\end{array}$ & 15 & $\begin{array}{l}\text { not } \\
\text { reported } \\
\text { to each } \\
\text { group }\end{array}$ & $\begin{array}{l}21.2 \\
(2.2)\end{array}$ & BDI-II & $24.30 \pm 11.90$ & $\begin{array}{c}20.00 \pm \\
12.00\end{array}$ & $\begin{array}{c}\text { Stretching } \\
\text { targeting } \\
\text { major muscle } \\
\text { groups }\end{array}$ & $30-45$ & 8 & 3 & not reported & $\begin{array}{c}\text { not } \\
\text { reported }\end{array}$ \\
\hline & $\begin{array}{l}\text { Intervention } \\
\text { group }\end{array}$ & 15 & $\begin{array}{l}\text { not } \\
\text { reported } \\
\text { to each } \\
\text { group }\end{array}$ & $\begin{array}{l}21.0 \\
(1.9)\end{array}$ & BDI-II & $24.50 \pm 11.50$ & $\begin{array}{c}10.00 \pm \\
10.00\end{array}$ & $\begin{array}{c}\text { Aerobic } \\
\text { continuous } \\
\text { steady-state } \\
\text { exercise }\end{array}$ & 45 & 8 & 3 & $\begin{array}{c}\text { 40-65\% of } \\
\text { HRR }\end{array}$ & $\begin{array}{c}\text { not } \\
\text { reported }\end{array}$ \\
\hline \multirow{2}{*}{$\begin{array}{c}\text { Roy, } \\
\text { Govindan } \\
\text { [39] }\end{array}$} & $\begin{array}{l}\text { Control } \\
\text { group }\end{array}$ & 20 & 100 & $\begin{array}{c}32.9 \\
(8.53)\end{array}$ & $\begin{array}{l}\text { HDRS- } \\
17\end{array}$ & $16.55 \pm 4.14$ & $\begin{array}{c}13.85 \pm \\
3.16\end{array}$ & TAU & - & - & - & - & $100 \%$ \\
\hline & $\begin{array}{l}\text { Intervention } \\
\text { group }\end{array}$ & 20 & 100 & $\begin{array}{c}31.1 \\
(8.88)\end{array}$ & $\begin{array}{c}\text { HDRS- } \\
17\end{array}$ & $19.30 \pm 4.83$ & $\begin{array}{l}15.00 \pm \\
4.93\end{array}$ & $\begin{array}{c}\text { Video } \\
\text { Assisted } \\
\text { Structured } \\
\text { Aerobic } \\
\text { Exercise } \\
\text { Program }\end{array}$ & 20 & 10 days & daily & $\begin{array}{l}\text { moderate to } \\
\text { low intensity }\end{array}$ & $100 \%$ \\
\hline $\begin{array}{l}\text { Siqueira, } \\
\text { Valiengo } \\
\text { [40] }\end{array}$ & $\begin{array}{l}\text { Control } \\
\text { group }\end{array}$ & 28 & 71 & $\begin{array}{l}37.86 \\
(9.85)\end{array}$ & $\begin{array}{l}\text { HDRS- } \\
17 \text { and } \\
\text { BDI }\end{array}$ & $\begin{array}{l}20.42 \pm 2.99 \\
33.92 \pm 9.64\end{array}$ & $\begin{array}{c}12.64 \pm \\
5.74 ; \\
20.50 \pm \\
10.21 \\
\text { Page } 8 / 2\end{array}$ & TAU & - & - & - & - & $\begin{array}{c}\text { not } \\
\text { reported }\end{array}$ \\
\hline
\end{tabular}




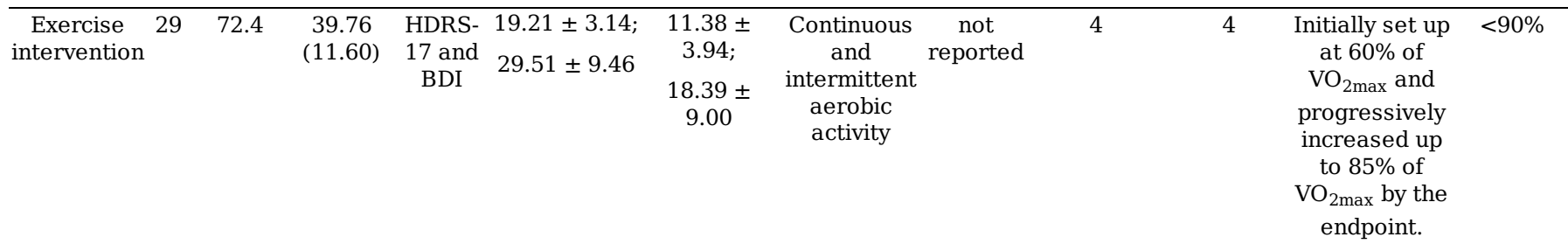

\begin{tabular}{|c|c|c|c|c|c|c|c|c|c|c|c|c|c|}
\hline \multirow[t]{2}{*}{$\begin{array}{c}\text { Strom, } \\
\text { Uckelstam } \\
{[41]}\end{array}$} & $\begin{array}{l}\text { Control } \\
\text { group }\end{array}$ & 24 & 83.3 & $\begin{array}{l}49.6 \\
(8.7)\end{array}$ & $\begin{array}{c}\text { MADRS } \\
\text { and } \\
\text { BDI-II }\end{array}$ & $\begin{array}{l}23.92 \pm 3.87 \\
28.25 \pm 7.08\end{array}$ & $\begin{array}{c}20.38 \pm \\
7.87 \\
24.04 \pm \\
6.86\end{array}$ & Wait list & - & - & - & - & - \\
\hline & $\begin{array}{c}\text { Intervention } \\
\text { group }\end{array}$ & 24 & 83.3 & $\begin{array}{c}48.8 \\
(12.7)\end{array}$ & $\begin{array}{l}\text { MADRS } \\
\text { and } \\
\text { BDI-II }\end{array}$ & $\begin{array}{l}23.54 \pm 4.39 \\
26.92 \pm 9.30\end{array}$ & $\begin{array}{c}15.71 \pm \\
7.54 ; \\
17.88 \pm \\
11.30\end{array}$ & $\begin{array}{l}\text { Aerobic self- } \\
\text { help programre } \\
\text { administered } \\
\text { through an } \\
\text { Internet- } \\
\text { based } \\
\text { system. }\end{array}$ & $\begin{array}{l}\text { not } \\
\text { ported }\end{array}$ & 9 & 3 & not reported & $\begin{array}{c}\text { not } \\
\text { reported }\end{array}$ \\
\hline
\end{tabular}

Abbreviations:

$\mathrm{N}=$ Total number of participants included in the study. Age = mean (standard deviation). Sex, F\% $=$ female percentual in the sample.

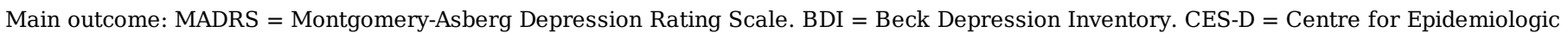
Studies Depression Scale. HDRS = Hamilton Depression Rating Scale.

Intervention: TAU = Usual care. CBT = Cognitive behaviour therapy. LD = Low dose. PHD = Public health dose.

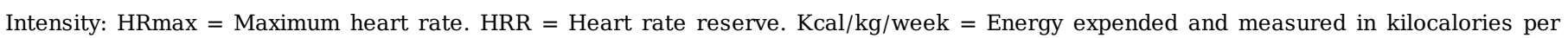
week. VO2max = Maximal oxygen consumption.

Adherence: percentage of finishers.

\subsection{Standardized mean difference (SMD)}

One study [30] presented four different intervention groups relative to the intensity and volume of exercise prescribed, which were included separately in the comparisons; two studies [32,37], which showed two different intervention groups associated with the presence of supervision or not and to the type of exercise, have also been included separately to ES analyses. SMD analyses in studies with more than one intervention group had the number of participants in the control group divided by the number of groups that existed for comparison in these studies. However, twelve studies [27-29, 31, 33-36, 38-41] showed just one intervention group to compare. To interpret SMD we used the scale suggested by Cohen [23]: >0.30 small; > 0.50 moderate; $>80$ large.

\subsubsection{Main analyses}

The forest plot of different treatments for depression in Figure 2 shows the global data with a small ES (SMD $=-0.45, \mathrm{Cl} 95 \%=-0.62$ to -0.29 , $\mathrm{P}$ $\left.<0.00001 ; \mathrm{T}^{2}=0.04, \mathrm{X}^{2}=0.08, \mathrm{I}^{2}=33 \%\right)$, and two subgroup analyses: exercise and pharmacological treatment $(\mathrm{SMD}=-0.70$ [moderate], $\mathrm{Cl} 95 \%$ $=-1.00$ to $\left.-0.40, P<0.00001 ; T^{2}=0.00, X^{2}=0.41, I^{2}=0 \%\right)$, and only exercise treatment (SMD $=-0.39$ [small], Cl95\% $=-0.57$ to $-0.21, P<0.0001$; $\mathrm{T}^{2}=0.04, \mathrm{X}^{2}=0.11, \mathrm{I}^{2}=31 \%$ ). The overall and subgroup analyses in different treatments for depression (ET and PT or only ET) have not shown heterogeneity among studies, with a trivial percentage of the variance. A visual analysis of the funnel plot in Figure 2 shows a symmetrical plot in the absence of publication bias.

\subsubsection{Subgroup analyses}

Forest and funnel plot of different characteristic analyses are displayed in the supplementary data, and Table 3 presents all of the subgroup SMD analyses with heterogeneity.

Global data in each feature of the selected studies showed: a small ES on exercise type (SMD $=-0.45, C l 95 \%=-0.59$ to $-0.31, P<0.00001 ; T^{2}=$ $\left.0.02, X^{2}=0.10, I^{2}=30 \%\right)$, and methods for intensity control $\left(S M D=-0.42, C 195 \%=-0.63\right.$ to $\left.-022, P<0.0001 ; T^{2}=0.05, X^{2}=0.10, I^{2}=33 \%\right)$, exercise frequency per week (SMD $=-0.45, \mathrm{Cl} 95 \%=-0.62$ to $\left.-0.29, \mathrm{P}<0.00001 ; \mathrm{T}^{2}=0.04, \mathrm{X}^{2}=0.08, \mathrm{I}^{2}=33 \%\right)$, intervention time $(\mathrm{SMD}=-0.47$, $\mathrm{Cl} 95 \%=-0.64$ to $-0.30, \mathrm{P}<0.00001 ; \mathrm{T}^{2}=0.04, \mathrm{X}^{2}=0.07, \mathrm{I}^{2}=35 \%$ ), intervention adherence $\left(\mathrm{SMD}=-0.41, \mathrm{Cl} 95 \%=-0.61\right.$ to $-0.22, \mathrm{P}<0.0001 ; \mathrm{T}^{2}=$ $0.05, X^{2}=0.05, I^{2}=41 \%$, age-range intervention ( $S M D=-0.46, C I 95 \%=-0.65$ to $-0.27, P<0.00001 ; T^{2}=0.06, X^{2}=0.06, I^{2}=35 \%$ ), control group characteristics (SMD $=-0.39, \mathrm{Cl} 95 \%=-0.59$ to $-0.21, \mathrm{P}<0.0001 ; \mathrm{T}^{2}=0.04, \mathrm{X}^{2}=0.11, \mathrm{I}^{2}=31 \%$ ), and a moderate ES on exercise duration per session (SMD $=-0.56, \mathrm{Cl} 95 \%=-0.74$ to $-0.37, \mathrm{P}<0.00001 ; \mathrm{T}^{2}=0.04, \mathrm{X}^{2}=0.10, \mathrm{I}^{2}=32 \%$ ). The overall analysis in different characteristics on the treatment for depression has not exhibited heterogeneity among studies, except on the intervention adherence. A visual analysis of the 
funnel plot in supplementary data did not show an asymmetrical plot, and also studies outside the pseudo-cone (CI 95\%), proving the absence of publication bias.

Table 3 shows that in favour of ET, with or without PT, the subgroup analysis on the treatment for depression has displayed : a small ES for two and three sessions $(p<0.00001)$, intervention time between one to nine weeks $(p=0.006), 50$ to $70 \%$ of intervention adherence ( $p=$ 0.0002), different age-range groups intervention at 30 to 60 years old ( $p=0.002)$ and 20 to 50 years old $(p=0.02)$, when compared to an active control group with stretching and light exercise ( $p=0.03)$; a moderate ES on resistance training $(p=0.02)$, moderate exercise intensity (60 to 80\%) controlled by MHR ( $p=0.0008)$, different exercise duration per session at 45 to $60 \mathrm{~min}(p<0.0001)$ and 20 to 30 min ( $<<0.0001)$, intervention time for more than 20 weeks ( $<0.00001)$, when compared to a control group with usual treatment use ( $p=0.0007)$; a large ES for seven sessions $(p=0.008)$, and intervention with people aged 60 years or more $(p<0.0001)$. The heterogeneity between studies in subgroup analysis was not exhibited, except on the moderate intensity exercise (60 to 80\%), exercise duration time for 45 to 60 min, intervention time between one to nine weeks, and intervention adherence between 50 to $70 \%$.

Table 3 - Subgroup meta-analysis. 


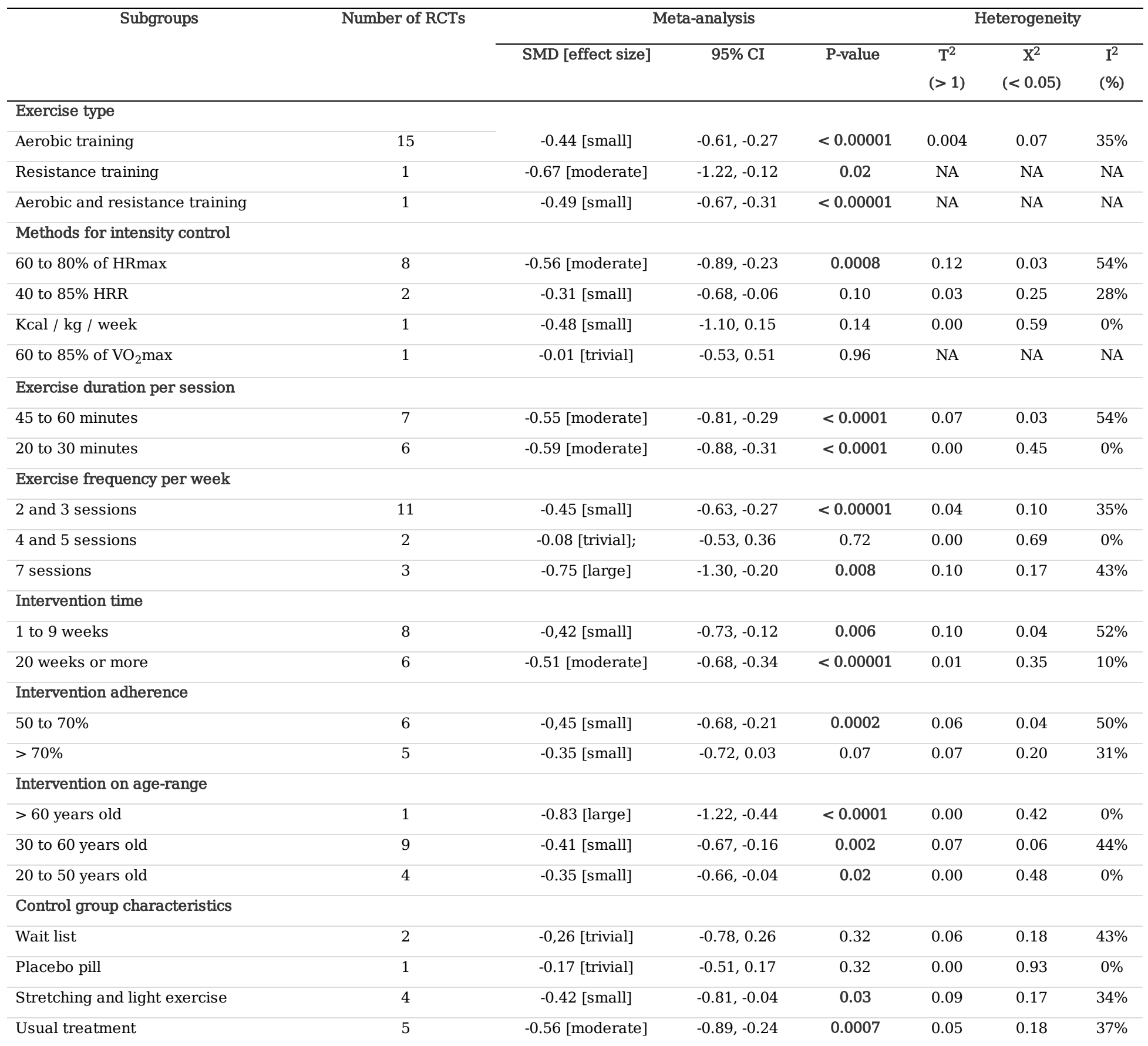

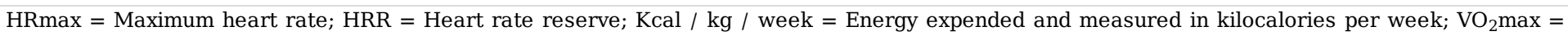

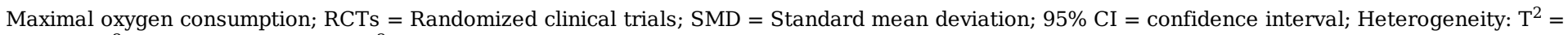
Tau $>1 ; X^{2}=$ Chi-value $(p<0.05) ; I^{2}=$ percentage of the variance; NA = Not applied, heterogeneity analyses with one subgroup selected;

\subsection{The gene network model in silico analyses}

Specific and non-specific (unknown) significant interactions (PPI Enrichment p-value <0.01) [15] among all genes were found in the network (Figure 4). Interactions were explored when specified edges were clearly shown, and the combined score reached 0.4 or above, determined as medium confidence (see String Statistics Section on https://string-db.org). Two genes (TPH2 and SLC6A4) have not met the criteria above and were excluded from the analysis. Therefore, the following genes remained significant (PPI Enrichment $p$-value $<0.03$ ) in the model: BDNF, HTR2A, APOE, IL6, INS, and TNF (Figure 5). The combined score of each interaction is shown in Table 4.

Table 4 - Specified interactions and confidence score of the gene network model 


\begin{tabular}{llll}
\hline Interaction & Type of interaction & Direction of interaction & Combined score \\
\hline HTR2A / BDNF & Unknown transcriptional regulation & Unidirectional & 0.80 \\
\hline APOE / BDNF & Unknow transcriptional negative regulation & Unidirectional & 0.81 \\
\hline IL-6 / BDNF & Unknown transcriptional regulation & Unidirectional & 0.81 \\
\hline TNF / BDNF & Unknown transcriptional negative regulation & Unidirectional & 0.80 \\
\hline IL-6 / APOE & Reaction and catalysis & Bidirectional & 0.96 \\
\hline INS / IL-6 & Unknown transcriptional regulation & Bidirectional & 0.94 \\
\hline TNF / IL-6 & Negative regulation and inhibition & Unidirectional & 0.98 \\
\hline APOE / INS & Binding & Unidirectional & 0.84 \\
\hline
\end{tabular}

\section{Discussion}

This study aimed to review the effect of additional ET, with or without PT, on the depression treatment. Moreover, its objective was to investigate subgroups in the selected studies to compare different exercise prescription, intervention, and control group characteristics, which could influence ET response. Finally, explore the hypothesis about the gene network model in silico analyses to explain how exercise could reduce depressive symptoms. After these analyses, results presented a moderate SMD on the additional effect of ET on PT for depression. Subgroup analyses showed that the moderate intensity, in 20 to 60-minute sessions preferably every day, could be the ideal exercise prescription in depressive treatment. Interventions prescribed 20 weeks or more with 50 to $70 \%$ of adherence may show a better result. When analysing based age-range studies, we found that ET can trigger a better result in older adults. Our gene network model also indicates that ET can be useful in decreasing depressive symptoms due to BDNF and inflammation interaction pathway, and regulation of HTR2A and APOE.

\subsection{Meta-analysis evidence}

This is the first review that confirms that exercise provides a vital add-on effect on PT for depression, according to the Gourgouvelis, Yielder [42] trial. It is not new that exercise training programs can be considered a treatment for depression and that PT is the gold-standard. However, antidepressants also promote a more rapid initial therapeutic response than exercise [43]. Considering this, it is adequate that ET and PT working together show a better result in the depression treatment.

There are preferences and growing evidence for using aerobic exercise in moderate-intensity to treat depression, as recent systematic reviews have shown $[8,12,14]$. Although, it is already clear that resistance training can produce a similar positive neurophysiological alteration by reducing depressive symptoms [44]. Even though one study, our review showed that resistance training could be a good alternative for the treatment. Based on the overload principle of training (progression), the exercise under a minimum intensity or threshold will not induce sufficiently the body to result in important neurophysiological adaptations for depression treatment [45]. In the initial program stage, a selfselected comfortable exercise intensity seems to be the better option to induce intrinsic exercise motivation and adherence [10]. We sustain that exercise intensity should be pleasurable, but it should also be gradually increased to guarantee a minimum level required to induce a training effect. Despite the diversity of tools to assess prescribed intensity (i.e., $\mathrm{HR}_{\text {max }}, \mathrm{HRR}, \mathrm{Kcal} / \mathrm{kg} /$ week, VO2 max , and the Borg scale), it is yet premature to provide recommendations concerning what is the better to control exercise intensity. In relation to the volume of prescribed exercise, other reviews [12-14] argue a reduced volume in the session duration and frequency when compared with our results. Utilizing the principle of progression again, we understand that the target in the volume prescription needs to follow our findings, which show a better response for both 20 to $30 \mathrm{~min}$ and 45 to 60 min every day. In the depression treatment, it does not matter if the patients are training for 20 or 60 minutes if they do it [46].

Regarding ET duration , past reviews reported positive outcomes after at least 8 or 9 weeks [12, 14]. However, based on the chronical neurobiological effects of exercise in depression, a regular exercise intervention is critical for satisfactory treatment response [47]. Corroborating that, our results show a better response for treatment of interventions equal to or more than 20 weeks. Intensity control contributes to the adherence in exercise intervention [10]. Notwithstanding, in the study by Callaghan, Khalil [48], the preferred intensity group only showed $66 \%$ of adherence. As well, when we observed data from studies where the preferred intensity was not prescribed but showed a significant reduction in depressive symptomatology, three studies [49-51] presented high rates of adherence (80-100\%). To our understanding, adherence rate is an essential factor for ET, although it resulted in a small effect in the present review. Adherence could be achieved not only by the most convenient intensity, but also with other strategies of intervention (e.g., knowledge of subjects' daily and leisure activities, exercise supervised and preferences about the context) [46]. 
It is essential to report that the effectiveness of pharmacological treatment in depression is not substantially affected by age [52]. According to a recent meta-analysis [53], exercise improves the response and can be considered as a routine tool in the management of depression in older adults. Noneless, Mura and Carta [54] have noticed the difficulty to establish the real effectiveness of exercise on depressive symptoms in elderly. However, the same author suggested that physical activity combined with antidepressants might be a beneficial strategy in treating late-life depression. The large ES showed in the older adult group in the current review can be related to the benefits that exercise promotes in other chronic diseases usually common in the elderly. Decreasing the symptoms of these comorbidities can result in a better socio-economic situation and positively impact on the quality of life of these depressive older adults [55].

Clinical trials that used an active control group, as well as usual treatment, must be carefully analysed because sometimes the results do not show reduced depressive symptoms when both groups are compared [11]. Researches [30,56] with stretching or yoga and light exercise in an active control group showed a significant reduction in the depressive symptomatology. It is not surprising since a meta-analysis concluded that yoga was an effective adjunct treatment of major psychiatric disorders, particularly depression and anxiety [57]. However, most often, there are improvements when the differences between baseline and post-intervention in each group are assessed, as in our study. When an exercise group is compared to an active control group instead of a passive control group, the differences between the efficacy of exercise and active placebos are much less pronounced. It is expected that doing something is likely to induce higher expectations for improvements than doing nothing [58]. Corroborating our viewpoint, a meta-analysis that investigated the predictors in control groups of exercise RCTs among adults with depression demonstrated that control group responses could negatively influence antidepressant efficacy [59].

\subsection{Bioinformatics analysis evidence}

Interesting molecular interactions among genes related to BDNF, serotonin receptor (5HTR2A), IL-6, INS, APOE, and TNF were identified in our protein-protein network model associated exercise. Literature has shown molecular interactions among these proteins in different conditions. BDNF and 5HT2A/2C agonists interact differently in the brain. While 5HT2A/2C agonists decrease BDNF on the hippocampus, they increase it dramatically in rats' neocortex and parietal cortex [60]. BDNF is also mediated by APOE isoforms. Sen, Nelson [61] demonstrated in a recent study that cultured human astrocytes responded differently when treated with APOE2, APOE3, and APOE4. BDNF was 19\% overexpressed in the astrocytes treated with APOE2 and 3, while APOE 4 decreased it $21 \%$ on average. Regarding inflammatory markers, IL-6 and TNF are proinflammatory cytokines that influence cell insulin uptake, causing insulin resistance [62, 63]. IL-6 and TNF-a are overexpressed in psychiatric disorders [64]. Decreased BDNF, serotonin deficits, APOE4 overexpression, increased pro-inflammatory cytokines and insulin resistance were already investigated as biomarkers of depression [63-67]. Furthermore, depressive patients show an atrophied hippocampus [68], which may be affected by decreased BDNF and increased cortisol. However, all of these biomarkers are modulated by exercise. For instance, IL- 6 is reduced in older adults who performed regular exercise (2-5 times, weekly) [69]. In addition, neurotransmitter synthesis and trophic factors secretion (e.g. BDNF) can improve serotoninergic, noradrenergic and dopaminergic circuitry through exercise [47, 70]. Hence, results from our gene network model suggest a clinical modulation of depressive symptoms by protein interactions through exercise.

\subsection{Limitations}

This systematic review has some limitations that must be considered. We included studies only since 2003, and this might have reduced the extent of the search scope. Concerning the missing data, selected studies in this current review do not report some essential data. Therefore, measures should be taken to improve the methodological standard of these RCT studies. Concerning heterogeneity, the random-effect model results in broader confidence intervals around the point estimates. However, it is a more conservative choice for the analysis [18]. Future research studies should evaluate exercise prescription reported in trials according to the application of the principles of exercise training (specificity, progression, overload, reversibility, diminishing returns). Also, studies should evaluate feelings of autonomous motivation in subjects with depression, as they are associated with the adherence and response to the treatment.

\section{Conclusion}

This study corroborated the additional effect of ET on PT for depression. We found that a long program with regular exercise has more effect on the treatment-response than the adherence percentage, and that ET can beneficiate older adults than adults and adolescents. Finally, the gene network model analyses involving molecular interactions in depression treatment showed biomarkers modulated by exercise, but this needs to be further explored.

\section{Abbreviations}

(DSM-IV) Diagnostic or Statistical Manual of Mental Disorders, Fourth Edition

$\% \mathrm{HR}_{\max } \quad$ Percentage of maximum heart rate 


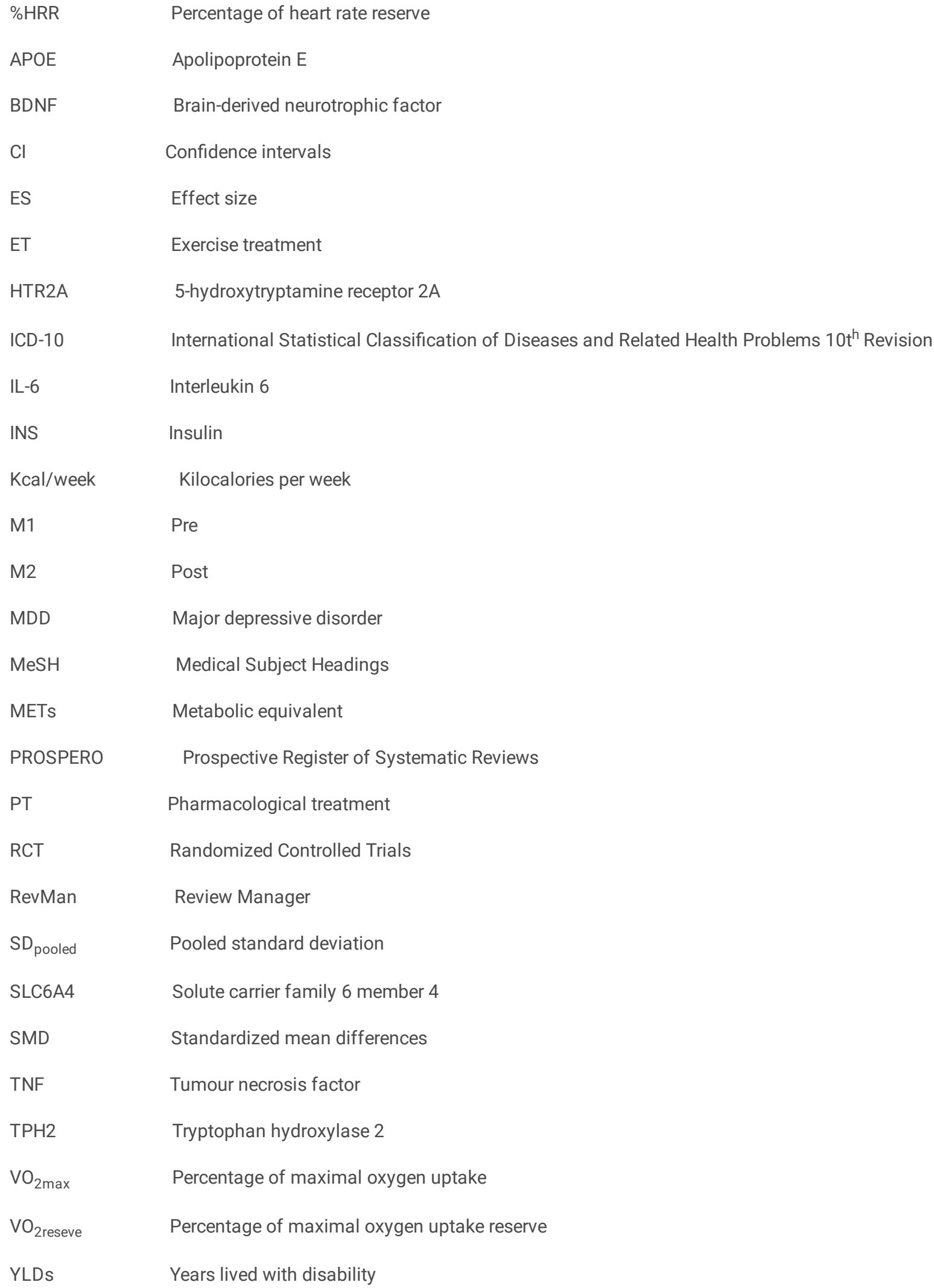

\section{Declarations}

\section{Ethics approval and consent to participate}

Not applicable.

\section{Consent to publish}


Not applicable.

\section{Availability of data and materials}

Data access statements, also known as data availability statements, are used in publications to describe where the data directly underpinning the publication can be found and under what conditions they can be accessed.

\section{Competing Interests}

The authors declare that they have no competing interests.

\section{Funding}

This work is financed by National Funds through FCT - Foundation for Science and Technology under the project UID04045 / 2020 . The founders had no intervention in any part of the study.

\section{Author's contributions}

Conception \& Design; LC, FS, MM, VR, RJ: Data analysis: LC, FS, HM, GA, RJ Interpretation of data; LC, FS, MM, MC, AD, HM, GA, RJ Draft and revising work LC, FS, MM; MC, AD, HM; GA, JR, RJ. ; All authors approved the final version.

\section{Acknowledgments}

Renato S. Monteiro-Junior thanks the Fundação de Amparo à Pesquisa do Estado de Minas Gerais (FAPEMIG) for a Post-doctoral support.

\section{References}

1. Zimmerman, M., T.A. Morgan, and K. Stanton, The severity of psychiatric disorders. World psychiatry : official journal of the World Psychiatric Association (WPA), 2018. 17(3): p. 258-275. doi: 10.1002/wps.20569.

2. Lepine, J.P. and M. Briley, The increasing burden of depression. Neuropsychiatr Dis Treat, 2011. 7(Suppl 1): p. 3-7. doi: 10.2147/NDT.S19617.

3. Kessler, R.C. and E.J. Bromet, The epidemiology of depression across cultures. Annu Rev Public Health, 2013. 34: p. 119-38. doi: 10.1146/annurev-publhealth-031912-114409.

4. Charlson, F., et al., New WHO prevalence estimates of mental disorders in conflict settings: a systematic review and meta-analysis. The Lancet, 2019. 394(10194): p. 240-248. doi.org/10.1016/S0140-6736(19)31141-9

5. Gelenberg, A.J., A review of the current guidelines for depression treatment. J Clin Psychiatry, 2010. 71(7): p.15. doi: 10.4088/JCP.9078tx1c.

6. Davidson, J.R., Major depressive disorder treatment guidelines in America and Europe. J Clin Psychiatry, 2010. 71 Suppl E1: p. 04. doi: 10.4088/JCP.9058se1c.

7. Ravindran, A.V., et al., Canadian Network for Mood and Anxiety Treatments (CANMAT) 2016 Clinical Guidelines for the Management of Adults with Major Depressive Disorder: Section 5. Complementary and Alternative Medicine Treatments. Can J Psychiatry, 2016. 61(9): p. 576-87. doi: 10.1177/0706743716660290.

8. Schuch, F.B., et al., Exercise as a treatment for depression: A meta-analysis adjusting for publication bias. J Psychiatr Res, 2016. 77: p. 4251. doi: 10.1016/j.jpsychires.2016.02.023.

9. Cooney, G.M., et al., Exercise for depression. Cochrane Database Syst Rev, 2013(9): p. CD004366. doi: 10.1002/14651858.CD004366.pub6.

10. Knapen, J., et al., Exercise therapy improves both mental and physical health in patients with major depression. Disabil Rehabil, 2015. 37(16): p. 1490-5. doi: 10.3109/09638288.2014.972579.

11. Ekkekakis, P., Honey, I shrunk the pooled SMD! Guide to critical appraisal of systematic reviews and meta-analyses using the Cochrane review on exercise for depression as example. Mental Health and Physical Activity, 2015. 8: p. 21-36. https://psycnet.apa.org/doi/10.1016/j.mhpa.2014.12.001.

12. Perraton, L.G., S. Kumar, and Z. Machotka, Exercise parameters in the treatment of clinical depression: a systematic review of randomized controlled trials. J Eval Clin Pract, 2010. 16(3): p. 597-604. doi: 10.1111/j.1365-2753.2009.01188.x

13. Nystrom, M.B., et al., Treating Major Depression with Physical Activity: A Systematic Overview with Recommendations. Cogn Behav Ther, 2015. 44(4): p. 341-52. doi: 10.1080/16506073.2015.1015440. 
14. Stanton, R. and P. Reaburn, Exercise and the treatment of depression: a review of the exercise program variables. J Sci Med Sport, 2014. 17(2): p. 177-82. doi: 10.1016/j.jsams.2013.03.010.

15. Franceschini, A., et al., STRING v9.1: protein-protein interaction networks, with increased coverage and integration. Nucleic Acids Res, 2013. 41(Database issue): p. D808-15. doi: 10.1093/nar/gks1094.

16. Akobeng, A.K., Principles of evidence based medicine. Arch Dis Child, 2005. 90(8): p. 837-40.

17. Moher, D., et al., Preferred reporting items for systematic reviews and meta-analyses: the PRISMA statement. PLoS Med, 2009. 6(7): p. e1000097. doi.org/10.1371/journal.pmed.1000097.

18. Higgins, J.P.T., and Green, S. , Cochrane Handbook for Systematic Reviews of Interventions 4.2.6. The Cochrane Library, 2006. Issue 4.

19. Higgins, J.P., et al., The Cochrane Collaboration's tool for assessing risk of bias in randomised trials. BMJ, 2011. 343: p. 1-9.

20. Huang, M., A. Neveol, and Z. Lu, Recommending MeSH terms for annotating biomedical articles. J Am Med Inform Assoc, 2011. 18(5): p. 660-7. doi: 10.1136/amiajnl-2010-000055.

21. Verhagen, A.P., et al., The Delphi list: a criteria list for quality assessment of randomized clinical trials for conducting systematic reviews developed by Delphi consensus. J Clin Epidemiol, 1998. 51(12): p. 1235-41. doi: 10.1016/s0895-4356(98)00131-0

22. Olivo, S.A., et al., Scales to assess the quality of randomized controlled trials: a systematic review. Phys Ther, 2008. 88(2): p. 156-75. doi.org/10.2522/ptj.20070147.

23. Cohen, J., Statistical power analysis for the behavioral sciences. 2nd ed. 1988, Hillsdale, N.J.: L. Erlbaum Associates.

24. Borenstein, M., Introduction to meta-analysis. 2009, Chichester, U.K.: John Wiley \& Sons. xxviii, 421 p.

25. Schuch, F.B., et al., Exercise and severe depression: preliminary results of an add-on study. J Affect Disord, 2011. 133(3): p. 615-8. doi: 10.1016/j.jad.2011.04.030.

26. de la Cerda, P., et al., Effect of an aerobic training program as complementary therapy in patients with moderate depression. Percept Mot Skills, 2011. 112(3): p. 761-9. doi.org/10.2466/02.15.PMS.112.3.761-769.

27. Buschert, V., et al., Effects of physical activity on cognitive performance: a controlled clinical study in depressive patients. Eur Arch Psychiatry Clin Neurosci, 2019. 269(5): p. 555-563. doi: 10.1007/s00406-018-0916-0.

28. Carneiro, L.S., et al., Effects of structured exercise and pharmacotherapy vs. pharmacotherapy for adults with depressive symptoms: $A$ randomized clinical trial. J Psychiatr Res, 2015. 71: p. 48-55. doi: 10.1016/j.jpsychires.2015.09.007.

29. Cheung, L.K. and S. Lee, A randomized controlled trial on an aerobic exercise programme for depression outpatients. Sport Sciences for Health, 2018. doi: 10.1002/da.22842.

30. Dunn, A.L., et al., Exercise treatment for depression: efficacy and dose response. Am J Prev Med, 2005. 28(1): p. 1-8. doi: 10.1016/j.amepre.2004.09.003

31. Hallgren, M., et al., Physical exercise and internet-based cognitive-behavioural therapy in the treatment of depression: randomised controlled trial. Br J Psychiatry, 2015. 207(3): p. 227-34. doi: 10.1192/bjp.bp.114.160101.

32. Hoffman, B.M., et al., Exercise fails to improve neurocognition in depressed middle-aged and older adults. Med Sci Sports Exerc, 2008. 40(7): p. 1344-52. doi: 10.1249/MSS.0b013e31816b877c

33. Kerling, A., et al., Effects of adjunctive exercise on physiological and psychological parameters in depression: a randomized pilot trial. J Affect Disord, 2015. 177: p. 1-6. doi: 10.1016/j.jad.2015.01.006.

34. Knubben, K., et al., A randomised, controlled study on the effects of a short-term endurance training programme in patients with major depression. Br J Sports Med, 2007. 41(1): p. 29-33. doi: 10.1136/bjsm.2006.030130

35. Krogh, J., et al., DEMO-II trial. Aerobic exercise versus stretching exercise in patients with major depression-a randomised clinical trial. PLoS One, 2012. 7(10): p. e48316. doi: 10.1371/journal.pone.0048316.

36. Legrand, F.D. and E.M. Neff, Efficacy of exercise as an adjunct treatment for clinically depressed inpatients during the initial stages of antidepressant pharmacotherapy: An open randomized controlled trial. J Affect Disord, 2016. 191: p. 139-44. doi: 10.1016/j.jad.2015.11.047.

37. Belvederi Murri, M., et al., Physical exercise for late-life major depression. Br J Psychiatry, 2015. 207(3): p. 235-42. doi: 10.1192/bjp.bp.114.150516.

38. Olson, R.L., et al., A randomized trial of aerobic exercise on cognitive control in major depression. Clin Neurophysiol, 2017. 128(6): p. 903913. https://doi.org/10.1016/j.clinph.2017.01.023.

39. Roy, A., R. Govindan, and K. Muralidharan, The impact of an add-on video assisted structured aerobic exercise module on mood and somatic symptoms among women with depressive disorders: Study from a tertiary care centre in India. Asian J Psychiatr, 2018. 32: p. 118122. doi: 10.1016/j.ajp.2017.12.004. 
40. Siqueira, C.C., et al., Antidepressant Efficacy of Adjunctive Aerobic Activity and Associated Biomarkers in Major Depression: A 4-Week, Randomized, Single-Blind, Controlled Clinical Trial. PLoS One, 2016. 11(5): p. e0154195. doi: 10.1371/journal.pone.0154195.

41. Strom, M., et al., Internet-delivered therapist-guided physical activity for mild to moderate depression: a randomized controlled trial. PeerJ, 2013. 1: p. e178. doi: 10.7717/peerj.178.

42. Gourgouvelis, J., et al., Exercise Leads to Better Clinical Outcomes in Those Receiving Medication Plus Cognitive Behavioral Therapy for Major Depressive Disorder. Front Psychiatry, 2018. 9: p. 37. doi: 10.3389/fpsyt.2018.00037.

43. Blumenthal, J.A., et al., Effects of exercise training on older patients with major depression. Arch Intern Med, 1999. 159(19): p. $2349-56$. https://doi.org/10.1001/archinte.159.19.2349.

44. Gordon, B.R., et al., Association of Efficacy of Resistance Exercise Training With Depressive Symptoms: Meta-analysis and Meta-regression Analysis of Randomized Clinical Trials. JAMA Psychiatry, 2018. 75(6): p. 566-576. doi: 10.1001/jamapsychiatry.2018.0572.

45. Brown, R., D. Ramirez, and J. Taub, The Prescription of Exercise for Depression. The Physician and sportsmedicine, $1978.6(12)$ : p. 34-45. doi.org/10.1080/00913847.1978.11710794.

46. Firth, J., et al., Motivating factors and barriers towards exercise in severe mental illness: a systematic review and meta-analysis. Psychol Med, 2016. 46(14): p. 2869-2881. doi: 10.1017/S0033291716001732.

47. Portugal, E.M., et al., Neuroscience of exercise: from neurobiology mechanisms to mental health. Neuropsychobiology, 2013. 68(1): p. 1-14.

48. Callaghan, P., et al., Pragmatic randomised controlled trial of preferred intensity exercise in women living with depression. BMC Public Health, 2011. 11: p. 465. doi: 10.1159/000350946.

49. Mota-Pereira, J., et al., Moderate exercise improves depression parameters in treatment-resistant patients with major depressive disorder. J Psychiatr Res, 2011. 45(8): p. 1005-11. doi: 10.1016/j.jpsychires.2011.02.005

50. Trivedi, M.H., et al., Exercise as an augmentation treatment for nonremitted major depressive disorder: a randomized, parallel dose comparison. J Clin Psychiatry, 2011. 72(5): p. 677-84. doi: 10.4088/JCP.10m06743.

51. Blumenthal, J.A., et al., Exercise and pharmacotherapy in the treatment of major depressive disorder. Psychosom Med, 2007. 69(7): p. 58796. doi: 10.1097/PSY.0b013e318148c19a

52. Frank, C., Pharmacologic treatment of depression in the elderly. Canadian family physician Medecin de famille canadien, 2014. 60(2): p. 121-126.

53. Schuch, F.B., et al., Exercise for depression in older adults: a meta-analysis of randomized controlled trials adjusting for publication bias. Braz J Psychiatry, 2016. 38(3): p. 247-54. doi: 10.1590/1516-4446-2016-1915.

54. Mura, G. and M.G. Carta, Physical activity in depressed elderly. A systematic review. Clinical Practice and Epidemiology in Mental Health, 2013. 9: p. 125-135. doi: 10.2174/1745017901309010125.

55. Lopez-Torres Hidalgo, J. and D.-E. Group, Effectiveness of physical exercise in the treatment of depression in older adults as an alternative to antidepressant drugs in primary care. BMC Psychiatry, 2019. 19(1): p. 21.

56. Chu, I., J. Buckworth, and T. Kirby, Effect of exercise intensity on depressive symptoms in women. Ment Health Phys Act, 2009. 2(1): p. 3743. doi: 10.1016/j.mhpa.2009.01.001

57. Cabral, P., H.B. Meyer, and D. Ames, Effectiveness of yoga therapy as a complementary treatment for major psychiatric disorders: a metaanalysis. Prim Care Companion CNS Disord, 2011. 13(4). doi: 10.4088/PCC.10r01068.

58. Stothart, C.R., et al., Is the effect of aerobic exercise on cognition a placebo effect? PLoS One, 2014. 9(10): p. e109557. doi.org/10.1371/journal.pone.0109557

59. Stubbs, B., et al., Challenges Establishing the Efficacy of Exercise as an Antidepressant Treatment: A Systematic Review and Meta-Analysis of Control Group Responses in Exercise Randomised Controlled Trials. Sports Med, 2016. 46(5): p. 699-713. doi: 10.1007/s40279-0150441-5.

60. Vaidya, V.A., et al., 5-HT\&lt;sub\&gt;2A\&lt;/sub\&gt; Receptor-Mediated Regulation of Brain-Derived Neurotrophic Factor mRNA in the Hippocampus and the Neocortex. The Journal of Neuroscience, 1997. 17(8): p. 2785.

61. Sen, A., T.J. Nelson, and D.L. Alkon, ApoE isoforms differentially regulates cleavage and secretion of BDNF. Molecular brain, 2017. 10(1): $\mathrm{p}$. 19-19. doi: 10.1186/s13041-017-0301-3.

62. Carey, A.L., et al., Interleukin-6 gene expression is increased in insulin-resistant rat skeletal muscle following insulin stimulation. Biochemical and Biophysical Research Communications, 2003. 302(4): p. 837-840. doi: 10.1016/s0006-291x(03)00267-5

63. Walsh, N.P., et al., Position statement. Part one: Immune function and exercise. Exerc Immunol Rev, 2011. 17: p. 6-63.

64. Dantzer, R., et al., From inflammation to sickness and depression: when the immune system subjugates the brain. Nat Rev Neurosci, 2008. 9(1): p. 46-56. doi: 10.1038/nrn2297

65. Gilmore, Walsh - 2008 - 10014_100-300Microcephalies and DNA repair.pdf>.

Page $17 / 20$ 
66. Wang, W.-W., et al., Depression was associated with apolipoprotein E $\varepsilon 4$ allele polymorphism: $A$ meta-analysis. Iranian journal of basic medical sciences, 2019. 22(2): p. 112-117. doi: 10.22038/ijbms.2018.30825.7436.

67. Santos, M.A.O., et al., Global hippocampal atrophy in major depressive disorder: a meta-analysis of magnetic resonance imaging studies. Trends in Psychiatry and Psychotherapy, 2018. 40: p. 369-378. doi: 10.1590/2237-6089-2017-0130.

68. Carvalho, Santos - 2014 - rof t o no it a c il b u p.pdf>.

69. Monteiro-Junior, R.S., et al., Effect of Exercise on Inflammatory Profile of Older Persons: Systematic Review and Meta-Analyses. Journal of Physical Activity and Health, 2018. 15(1): p. 64-71. doi: 10.1123/jpah.2016-0735.

70. Lin, T.W. and Y.M. Kuo, Exercise benefits brain function: the monoamine connection. Brain Sci, 2013. 3(1): p. 39-53. doi: 10.3390/brainsci3010039.

\section{Figures}

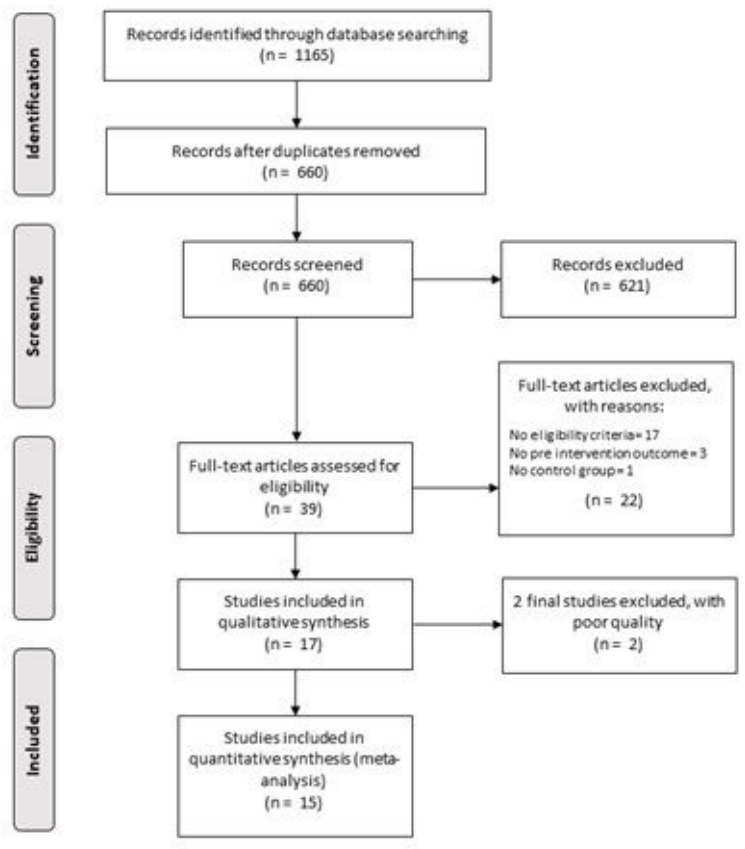

Figure 1

Flow-chart of the selected studies.

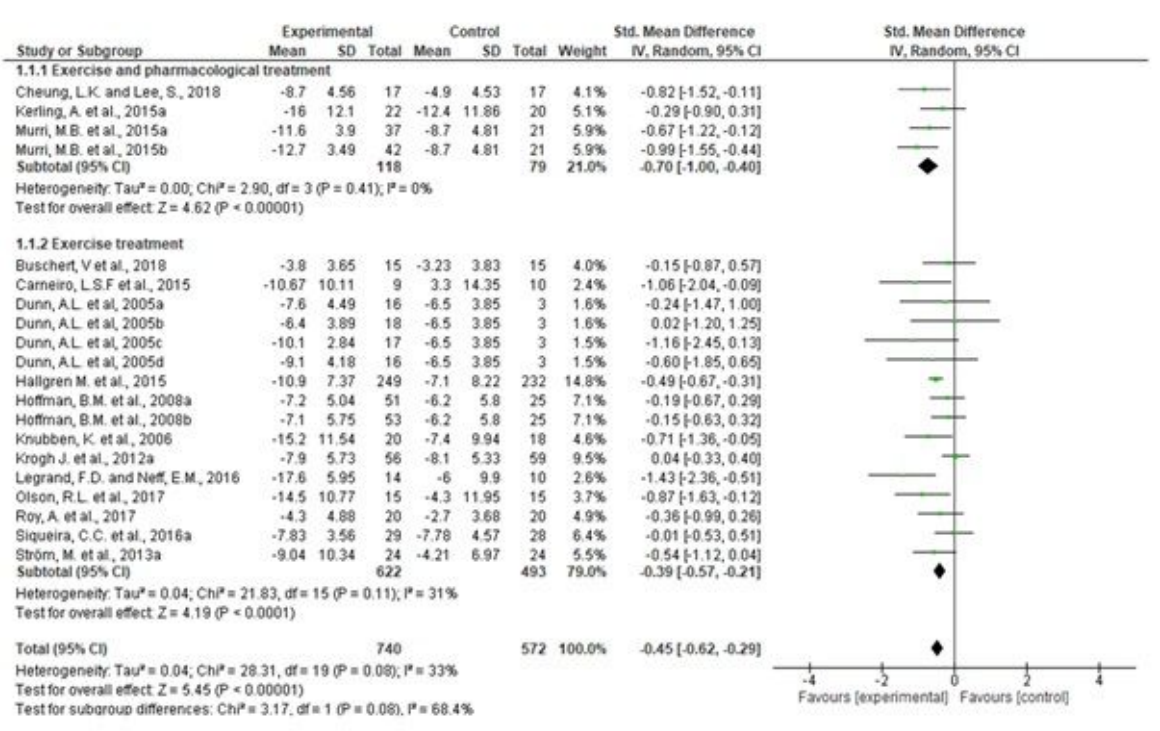


Forest plot.

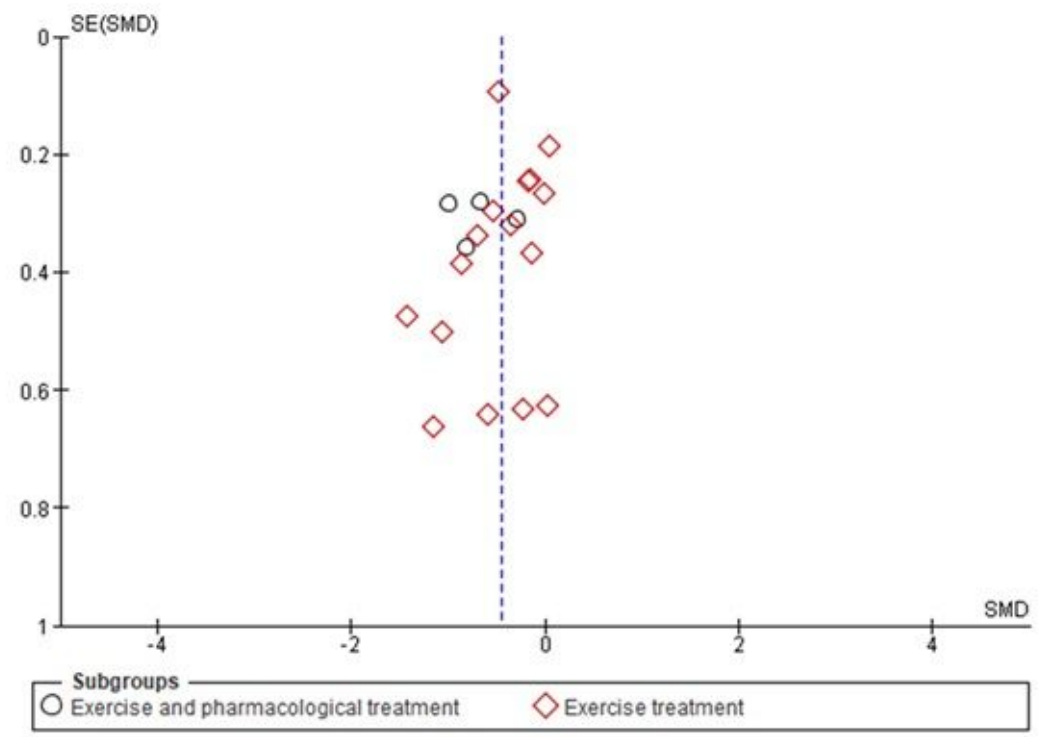

\section{Figure 3}

Funnel plot.

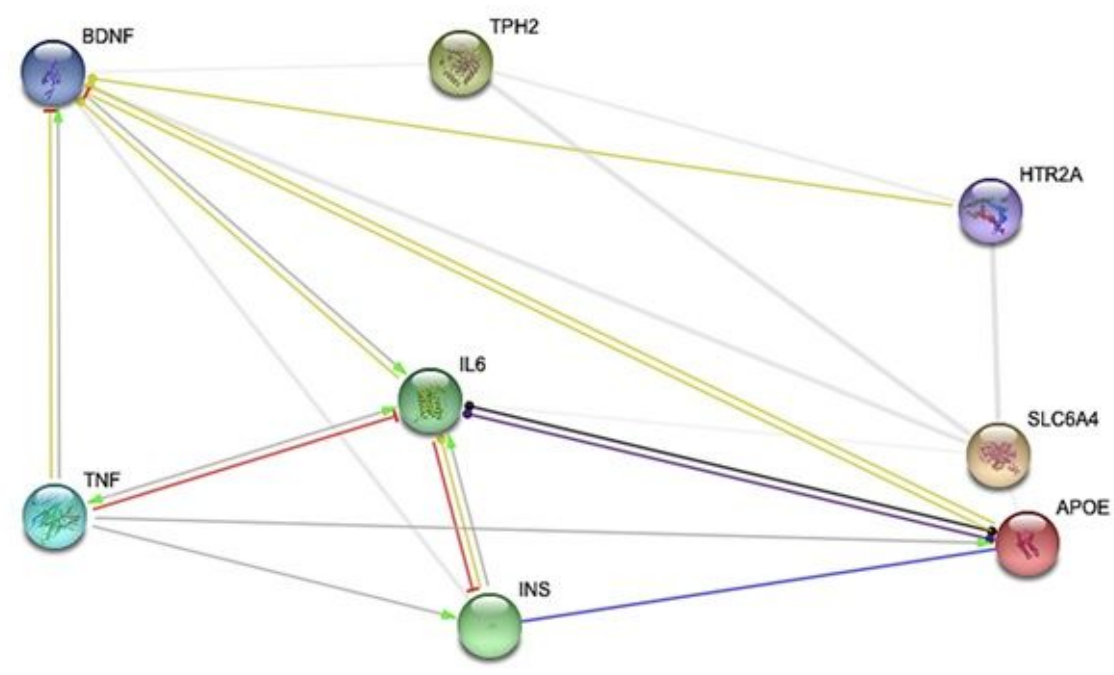

\section{Figure 4}

Full network genes interaction. Coloured lines (yellow, blue, red, purple, and black): known interactions; Grey lines: unknown interactions; Arrow: positive action; Dots: unspecified interaction; Dash: negative action. 


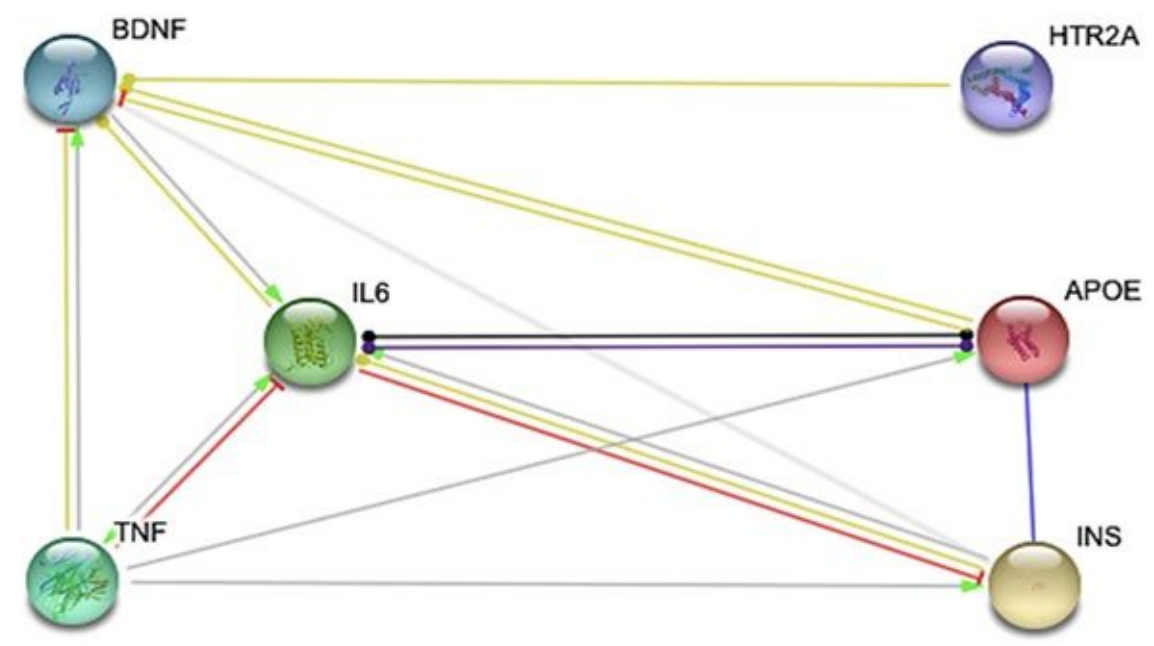

\section{Figure 5}

Clear network genes interaction. Yellow line: transcriptional regulation; Red line: inhibition; Black line: reaction; Purple line: catalysis; Blue line: binding; Grey lines: unknown interactions; Arrow: positive action; Dots: unspecified interaction; Dash: negative action.

\section{Supplementary Files}

This is a list of supplementary files associated with this preprint. Click to download.

- supplement1.docx 